Check for updates

Cite this: RSC Adv., 2018, 8, 15119

\section{The investigation of the specific behavior of a cationic block structure and its excellent flocculation performance in high-turbidity water treatment $\uparrow$}

\author{
Li Feng, (D) ab Huaili Zheng, ${ }^{\text {ab }}$ Xiaomin Tang, ${ }^{\text {bc }}$ Xinyu Zheng, ${ }^{\text {b }}$ Shuang Liu, ${ }^{\text {ab }}$ \\ Qiang Sun ${ }^{\mathrm{b}}$ and Moxi Wang ${ }^{\mathrm{b}}$
}

The fabrication of a cationic polyacrylamide (CPAM) with high efficiency and economy has been highly desired in the field of high-turbidity water treatment. This study introduced an ultrasound (US)-initiated template polymerization (UTP) method to develop a novel cationic templated polyacrylamide (TPAA) with a microblock structure. TPAA was prepared using acrylamide (AM) and sodium (3-acrylamidopropyl) trimethylammonium chloride (ATAC) as the monomers and sodium polyacrylate (NaPAA) as the template. Factors that affected polymerization such as the ultrasound power, ultrasound time, initiator concentration, $\mathrm{pH}$, and $m_{\mathrm{AM}}: m_{\mathrm{ATAC}}$ and $n_{\mathrm{NaPAA}}: n_{\mathrm{ATAC}}$ values were investigated. The properties of the polymers were characterized by Fourier transform infrared spectroscopy (FTIR), ${ }^{1} \mathrm{H}$ nuclear magnetic resonance spectroscopy $\left({ }^{1} \mathrm{H}\right.$ NMR), thermogravimetric analysis (TGA) and scanning electron microscopy (SEM). The results indicated the successful formation of a cationic microblock structure in TPAA. In addition, TPAA displayed favorable thermal decomposition properties and a rough and coarse surface morphology, as shown by analyses using TGA and SEM, respectively. Moreover, a zip (type I) template polymerization mechanism was identified via analyses of the association constant $\left(K_{M}\right)$, conversion $\left(C_{v}\right)$ and polymerization rate $\left(R_{\mathrm{p}}\right)$. The flocculation performance of the templated copolymer TPAA was evaluated by treating high-turbidity water. According to the results for the zeta potentials and FTIR spectra of the generated flocs, it was indicated that the cationic microblocks in the templated copolymer could greatly enhance its charge neutralization, patching and bridging ability, and therefore excellent flocculation performance (residual turbidity: 5.8 NTU, $D_{\mathrm{f}}: 1.89$, floc size $d_{50}: 608.404 \mu \mathrm{m}$ and floc kinetic: $15.86 \times 10^{-4} \mathrm{~s}^{-1}$ ) for treating high-turbidity water was achieved.
Received 7th March 2018 Accepted 7th April 2018

DOI: $10.1039 / c 8 \mathrm{ra02006j}$

rsc.li/rsc-advances and river blockages. ${ }^{3,4}$ Moreover, high-turbidity wastewater will block filter holes and abrade water treatment equipment in water purification plants and thus reduce the efficiency of treatment or even paralyze the treatment process. Therefore, the development of an efficient and economical method to treat highly turbid water is an important issue for environmental engineers. ${ }^{5}$ Fortunately, the flocculation method has attracted much attention owing to its high efficiency, low cost, convenient operation and enhancement of its functions via modification.

Cationic polyacrylamide (CPAM) has been developed and \footnotetext{
widely used for the separation and treatment of high-turbidity water because of its satisfactory solid-water separation performance and the negative charge properties of most highturbidity wastewater. ${ }^{6,7}$ Negatively charged particles can be thoroughly neutralized and destabilized by the cationic chemical group, and then these destabilized particles will be captured by the molecular chain of the polymer to agglomerate and form large and compact flocs. ${ }^{8}$ As a result, these particles

${ }^{a}$ State Key Laboratory of Coal Mine Disaster Dynamics and Control, Chongqing University, Chongqing 400044, China. E-mail: hlz6512@163.com; Fax: +86 23 65120827; Tel: +862365120827

${ }^{b}$ Key Laboratory of the Three Gorges Reservoir Region's Eco-Environment, Ministry of Education, Chongqing University, Chongqing 400045, China

${ }^{c}$ Chongqing Key Laboratory of Catalysis and Environmental New Materials, College of Environment and Resources, Chongqing Technology and Business University, Chongqing 400067, P. R. China

$\dagger$ Electronic supplementary information (ESI) available. See DOI: $10.1039 / \mathrm{c} 8 \mathrm{ra02006j}$
} 
are thoroughly separated and removed from the water. Thus far, various CPAMs and their modified forms have been successfully synthesized for use in heavy metal removal, oil mill effluent treatment, kaolin wastewater removal, highturbidity water separation and so on. ${ }^{9-12}$ These flocculants perform well and exhibit excellent flocculation performance for the removal and separation of particles. Although progress and triumphs in the design and development of polymers have been achieved, great efforts are still needed to design new economic and efficient flocculants or propose new ways to overcome bottlenecks in existing methods for preparing novel flocculants. Template synthesis technology has become increasingly attractive for synthesizing nanotubes, nanopore arrays, and nanostructures owing to its controllable and tunable advantages. ${ }^{\mathbf{1 3 - 1 6}}$ In order to obtain the desired products, a template with a specific and novel structure is used to construct the skeleton, and the materials used for the products will combine with the template to form precursors of the desired products via electrostatic attraction, hydrogen bonding interactions and chemical/physical effects. ${ }^{17}$ When the template is separated, the remaining materials will have the same desired structure as that of the template, and thus this technology is so powerful. If template synthesis technology is introduced and used to develop flocculants with novel structures, the flocculation ability of the flocculants will undergo a great improvement. As shown in Fig. 1, the novel microblock structure in the polymer chain will have a strong electrostatic charge force and high charge efficiency to neutralize negatively charged particles completely to form a more compact floc structure, whereas a random distribution of charges in the polymer chain will lead to poor flocculation performance. However, there have been few reports of the use of this new template synthesis technology in the design and development of polymers, and hence it will be more meaningful and constructive to use this method for the preparation of polymers.

(3-Acrylamidopropyl)trimethylammonium chloride (ATAC) and acrylamide (AM) are common monomers used for the preparation of polymers, and their products have been widely used in the removal of high-turbidity water. ${ }^{18,19}$ Therefore, ATAC and AM were selected as the monomers to prepare the

(a)

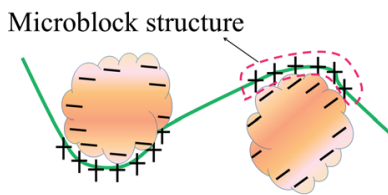

(b)

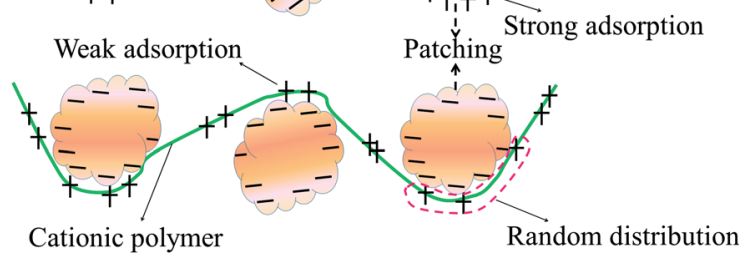

Fig. 1 Flocculation models for (a) templated copolymer with microblock structure and (b) random distributed copolymer. templated copolymer TPAA with the novel microblock structure by template polymerization, as this would be more significant and challenging. Sodium polyacrylate (NaPAA) was chosen as the template in this experiment because its molecular weight is tunable and it is easily separated with ethyl alcohol. Moreover, ultrasound (US)-initiated polymerization is a popular topic owing to its advantages such as low cost, easy operation, high efficiency and environmental safety. ${ }^{20,21}$ It has also been reported that US-initiated polymerization has a specific function in modifying the polymer surface and obtaining a more porous and coarse surface morphology, which thus improves the dissolution of the polymer and enhances its flocculation performance. ${ }^{22}$ On the basis of the merits of US-initiated polymerization, US-initiated template polymerization technology should be adopted to prepare the flocculant TPAA with the novel microblock structure.

In view of all the aforementioned aspects, this study aimed to: (1) synthesize the templated copolymer (TPAA) with the cationic microblock structure by US-initiated template polymerization technology using ATAC and AM as the monomers and NaPAA as the template; (2) examine the synthesis conditions, namely, the ultrasound power, ultrasound time, initiator concentration, $\mathrm{pH}$, and $m_{\mathrm{AM}}: m_{\mathrm{ATAC}}$ and $n_{\mathrm{NaPAA}}: n_{\mathrm{ATAC}}$ values, depending on the intrinsic viscosity and conversion; (3) determine the template polymerization mechanism by investigating the association constant $\left(K_{\mathrm{M}}\right)$, conversion $\left(C_{\mathrm{v}}\right)$ and polymerization rate $\left(R_{\mathrm{p}}\right)$; (4) characterize the structural properties of the polymers by employing FTIR, ${ }^{1} \mathrm{H}$ NMR, TGA and scanning electron microscopy (SEM); (5) evaluate the flocculation performance of the polymers in terms of residual turbidity, floc size, recoverability, fractal dimension and settling behaviors; and (6) discuss the flocculation mechanism via analyses of the zeta potentials and FTIR spectra of the formed flocs.

\section{Experimental section}

\subsection{Materials}

Acrylamide (AM) was a gift from Lanjie Tap Water Company (Chongqing, China) and was of industrial grade. (3-Acrylamidopropyl)trimethylammonium chloride (ATAC) (74-76 wt\% in water, industrial grade) was obtained from Longsheng Chemical Co., Ltd (Shanghai, China). Sodium polyacrylate (NaPAA) with a molecular weight (MW) of 5300 and the initiator 2,2'-azobis[2-(2-imidazolin-2-yl)propane] dihydrochloride (VA044) were both purchased from Apotheker Chemical Reagent Co., Ltd (Chendou, China) and were both of analytical grade. In order to carry out a controlled trial, the commercial flocculants CCPDA and CCPMA were purchased from Lanjie Tap Water Company (Chongqing, China). CCPDA was prepared from AM and acryloyloxyethyltrimethylammonium chloride (DAC), and CCPMA was formed by copolymerization of AM and methacryloxyethyltrimethylammonium chloride (DMC). Besides, polyacrylamide (PAM) made in the laboratory was used for the characterization analysis. 


\subsection{Synthesis of copolymers}

Possible routes for the US-initiated synthesis of the templated copolymer TPAA and randomly distributed copolymer CPAA are illustrated in Fig. 2, and the procedure for the preparation of the templated copolymer TPAA was as follows: predetermined doses of AM, ATAC, NaPAA $\left(n_{\mathrm{NaPAA}}: n_{\mathrm{ATAC}}=1: 1\right)$ and deionized water were added to a $100 \mathrm{~mL}$ glass vessel, and the vessel was shaken several times until the reagents dissolved. The total monomer concentration was fixed at $30 \%$. Then, the $\mathrm{pH}$ of the above aqueous solution was adjusted to the set value with $0.5 \mathrm{~mol} \mathrm{~L}^{-1} \mathrm{HCl}$ and $\mathrm{NaOH}$. Subsequently, nitrogen was bubbled into the solution for $30 \mathrm{~min}$, and the vessel was sealed immediately after the addition of the initiator VA-044. After the above procedures were performed, the solution was subjected to ultrasonic radiation generated by an ultrasound generator (Sonics Vibra-Cell VCX 500; power: 0-500 W; frequency: $20 \mathrm{kHz}$; horn type; sonic wave probe: tip diameter $=13 \mathrm{~mm}$, length $=$ $136 \mathrm{~mm}$ ) for $15 \mathrm{~min}$ at room temperature to finish the copolymerization reaction. Finally, the solid product was purified with ethyl alcohol and desiccated in a vacuum oven at $95^{\circ} \mathrm{C}$ for $24 \mathrm{~h}$ to obtain the final product. On the other hand, the method for the preparation of the randomly distributed copolymer CPAA was similar to that used for TPAA, and the only difference was that no NaPAA was added for CPAA. In addition, polyacrylamide (PAM) was prepared by the above method (US-initiated polymerization) to perform a comparative characterization of the polymers.

\subsection{Characteristics of copolymers}

According to previous research, the total monomer conversion and the intrinsic viscosities of the polymers $(\eta)$ were measured by the gravimetric and one-point methods, respectively. ${ }^{1,23}$ The positive charge density of the polymers, which was used to evaluate their charge neutralization ability, was measured by a titration method. ${ }^{24}$ Poly(vinyl sulfate) potassium salt (PVSK) and toluidine blue $\mathrm{O}$ (TBO) were used as the titrant and indicator, respectively.

Instrumental analyses such as FTIR and ${ }^{1} \mathrm{H}$ NMR spectroscopy of the polymers were performed with a 550 Series II infrared spectrometer (Mettler Toledo Instruments Co., Ltd, Switzerland) using $\mathrm{KBr}$ pellets and an Avance 500 nuclear magnetic resonance spectrometer (Bruker Company, Ettlingen, Germany) using deuterium oxide $\left(\mathrm{D}_{2} \mathrm{O}\right)$ as the solvent, respectively. The morphologies of the copolymers were recorded with a MIRA 3 LMU SEM system (TESCAN Company, Czech Republic). Thermogravimetric analysis (TGA) was conducted with a DTG-60H simultaneous thermal analyzer (Shimadzu, Kyoto, Japan) at a heating rate of $10{ }^{\circ} \mathrm{C} \mathrm{min}^{-1}$ and a nitrogen flow rate of $20 \mathrm{~mL} \mathrm{~min}^{-1}$ in the temperature range of $20-600^{\circ} \mathrm{C}$.

\subsection{Determination of $K_{\mathrm{M}}, C_{\mathrm{v}}$ and $R_{\mathrm{p}}$}

On the basis of previous studies, the association constant $\left(K_{\mathrm{M}}\right)$ can be used to examine the strength of the interaction between the template NaPAA and the cationic monomer and also the mechanism of template polymerization. ${ }^{25,26}$ Besides, measurements of the conversion $\left(C_{\mathrm{v}}\right)$ and polymerization rate $\left(R_{\mathrm{p}}\right)$ are also important and helpful for understanding the template polymerization mechanism and the effect of the template on the polymerization. In this study, dialysis and conductometric titration methods were used to determine the values of $K_{\mathrm{M}}, C_{\mathrm{v}}$ and $R_{\mathrm{p}}$, and details of these methods are displayed in ESI S1. $\dagger$

\subsection{Flocculation experiments}

2.5.1. Jar tests. Jar tests were conducted using a programcontrolled jar test apparatus (ZR4-6, Zhongrun Water Industry Technology Development Co. Ltd, China) with a six-place programmed paddle mixer at room temperature. A kaolin suspension $\left(0.5 \mathrm{~g} \mathrm{~L}^{-1}\right)$ with an original turbidity of $143 \mathrm{NTU}$ and an original zeta potential of $-43.7 \mathrm{mV}$ at a $\mathrm{pH}$ of 7 was used as simulated high-turbidity wastewater. The kaolin used in this experiment was characterized by an average size of $3.5 \mu \mathrm{m}$, a density of $2.6 \mathrm{~g} \mathrm{~cm}^{-3}$ and a purity of $98 \%$. Coagulation experiments were also performed at different $\mathrm{pH}$ values, and the $\mathrm{pH}$ was adjusted using appropriate volumes of $0.1 \mathrm{~mol} \mathrm{~L}^{-1} \mathrm{HCl}$ and $\mathrm{NaOH}$. The detailed procedure of the jar tests comprised rapid stirring at $200 \mathrm{rpm}$ for $5 \mathrm{~min}$ to ensure sufficient mixing of the flocculants with the wastewater, slow stirring at $50 \mathrm{rpm}$ for $15 \mathrm{~min}$ for the growth of flocs, and finally a settling period of $30 \mathrm{~min}$ for floc sedimentation. After the completion of these steps, samples were collected from the supernatants for further measurements of the residual turbidity using a 2100P turbidity meter (Hach, Loveland, CO), zeta potential (ZP) measurements

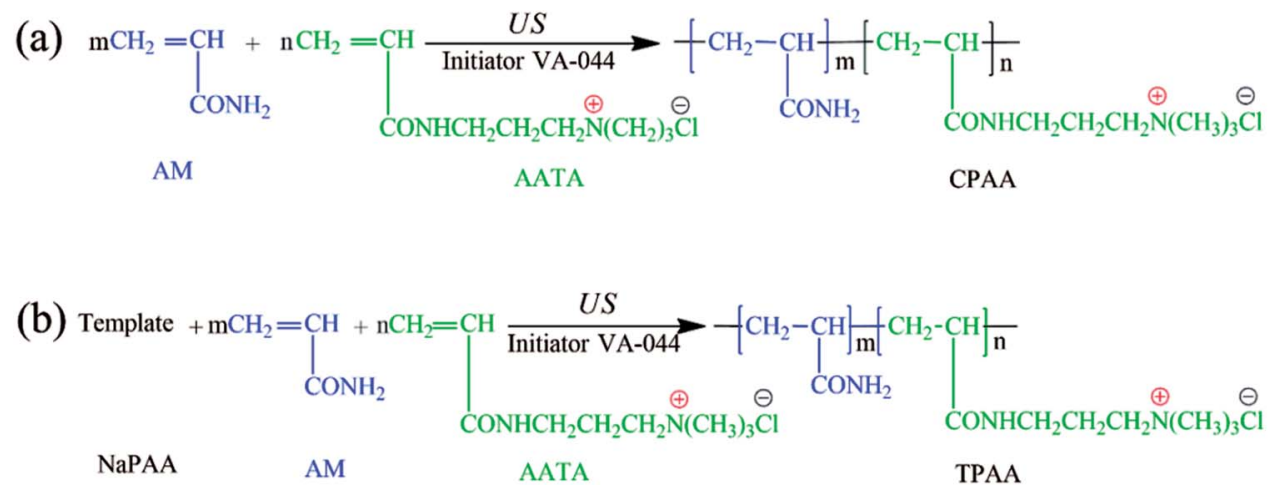

Fig. 2 Possible routes for the US-initiated synthesis of (a) the randomly distributed copolymer CPAA and (b) the templated copolymer TPAA. 
Table 1 Details of flocculants used for the characterization and jar tests

\begin{tabular}{|c|c|c|c|c|}
\hline Flocculant $^{a}$ & $\begin{array}{l}\text { Cationic monomer } \\
\text { molar content }(\%)\end{array}$ & $\begin{array}{l}\text { Intrinsic viscosity } \\
\left(\mathrm{dL} \mathrm{g}^{-1}\right)\end{array}$ & $\begin{array}{l}\text { Conversion } \\
\text { rate }(\%)\end{array}$ & $\begin{array}{l}\text { Synthetic } \\
\text { method }\end{array}$ \\
\hline TPAA & 30.0 & 8.49 & 99.7 & UTP \\
\hline CCPDA & 30.0 & 8.51 & - & - \\
\hline CCPMA & 30.0 & 8.51 & - & - \\
\hline PAM & - & 8.50 & 99.8 & N-UTP \\
\hline
\end{tabular}

${ }^{a}$ TPAA: templated copolymer of ATAC and AM produced by US-initiated template copolymerization (UTP); CPAA: copolymer of ATAC and AM produced by non-US-initiated template copolymerization (N-UTP); PAM: homopolymer of AM produced by N-UTP; CCPDA: commercial copolymer of DAC and AM; CCPMA: commercial copolymer of DMC and AM.

using a Zetasizer Nano ZS90 analyzer (Malvern Instruments Ltd, Malvern, UK) and floc size analysis using a Zetasizer 3000HSA (Malvern Instruments, UK), respectively. Each result recorded under the corresponding conditions was the average of three repeated measurements, and the standard deviation was controlled at less than $5 \%$. Details of the flocculants used for the characterization and jar tests are shown in Table 1. Besides, the flocculation kinetics of the four flocculants was also investigated using eqn (1), which is expressed as follows:

$$
\left(\frac{N_{0}}{N_{t}}\right)^{\frac{1}{2}}=1+0.5 \times k N_{0} t
$$

where $N_{0}$ is the initial turbidity (143 NTU), $N_{t}$ is the turbidity as a function of time $(1,4,7,11,14,17$, and $20 \mathrm{~min})$ after slow stirring (50 rpm for $15 \mathrm{~min}$ ) during each flocculation test, and $k$ is the kinetic constant of the flocculation system.

2.5.2. Determination of fractal dimension $\left(D_{\mathrm{f}}\right)$. Fractal geometry has been widely used to describe the structure of particles, and the aggregate structure can be simply described by the fractal dimension $\left(D_{\mathrm{f}}\right) \cdot{ }^{27,28} D_{\mathrm{f}}$ is an important index that represents the degree of compactness of the primary particles and was used to evaluate the solid/liquid separation efficiency. In this experiment, the $D_{\mathrm{f}}$ value of the kaolin floc that formed was measured after the settling period. Details of the $D_{\mathrm{f}}$ measurements are available in ESI S2.†

\section{Results and discussion}

\subsection{Optimizing synthesis conditions}

3.1.1. Effect of ultrasound power on copolymerization. The ultrasound waves, which are regarded as the core component of the US-initiated polymerization system, released more energy to promote molecular collisions and accelerate the cleavage of the initiator to form more free radicals and thereby initiated the copolymerization of the monomers. Therefore, the effect of the ultrasound power on copolymerization was investigated at an ultrasound time of $15 \mathrm{~min}$, an initiator concentration of 0.06 $\mathrm{wt} \%, \mathrm{pH}=4.5, m_{\mathrm{AM}}: m_{\mathrm{ATAC}}=3: 1$ and $n_{\mathrm{NaPAA}}: n_{\mathrm{ATAC}}=1: 1$. As depicted in Fig. 3(a), it was shown that an ultrasound power of $200 \mathrm{~W}$ was more favorable for copolymerization, whereas an ultrasound power of higher or lower than $200 \mathrm{~W}$ was unsatisfactory for copolymerization. At a lower ultrasound power, the initiation energy was not sufficiently high to generate enough free radicals, and the collision of monomers was inefficient. As a result, the copolymerization efficiency was reduced, which thus led to decreases in the intrinsic viscosity and conversion. In contrast, a higher ultrasound power promoted the cleavage of the initiator to generate superfluous free radicals within a short time and also resulted in violent collisions of monomers. Thus, chain transfer and chain termination accelerated, and decreases in intrinsic viscosity and conversion occurred. ${ }^{29}$ Besides, the stronger shear force caused by the swelling and collapse of cavities destroyed and fragmented the polymer chains that formed, which thus led to low intrinsic viscosity. Accordingly, the favorable ultrasound power of $200 \mathrm{~W}$ was used in this study to achieve high-efficiency copolymerization.

3.1.2. Effect of ultrasound time on copolymerization. As shown in Fig. 3(c), the effect of varying the US irradiation time on polymerization was evaluated while the other factors were kept constant. It was obvious that the intrinsic viscosity and conversion increased rapidly with an increase in the US irradiation time from $10 \mathrm{~min}$ to $15 \mathrm{~min}$, whereas almost no increases in the intrinsic viscosity and conversion were observed when the US irradiation time was more than $15 \mathrm{~min}$. The US radiation would decompose the initiator and generate free radicals to accelerate the breaking of molecular bonds and increase the number of grafting sites and thus lead to rapid increases in the intrinsic viscosity and conversion. ${ }^{30}$ However, the initiator would disintegrate and be used up under continued US radiation ( $>15 \mathrm{~min}$ ), and no free radicals would be generated any longer. Consequently, the intrinsic viscosity and conversion maintained an unchanged trend when the irradiation time exceeded $15 \mathrm{~min}$. Therefore, $15 \mathrm{~min}$ was chosen as the optimal irradiation time in this study.

3.1.3. Effect of initiator dosage on copolymerization. Fig. 3(b) shows the effect of varying the initiator dosage on the intrinsic viscosity and conversion while keeping the other factors constant. The intrinsic viscosity and conversion increased initially with an increase in the initiator dosage from $0.01 \mathrm{wt} \%$ to $0.06 \mathrm{wt} \%$ and then declined rapidly with a further increase in the initiator concentration. The few free radicals generated by a lower initiator dosage would be surrounded by a large number of solvent molecules, resulting in a "cage effect", which would prevent the formation of monomeric free radicals and further chain growth. ${ }^{31}$ A relatively higher initiator dosage was favorable for increasing the intrinsic viscosity and 

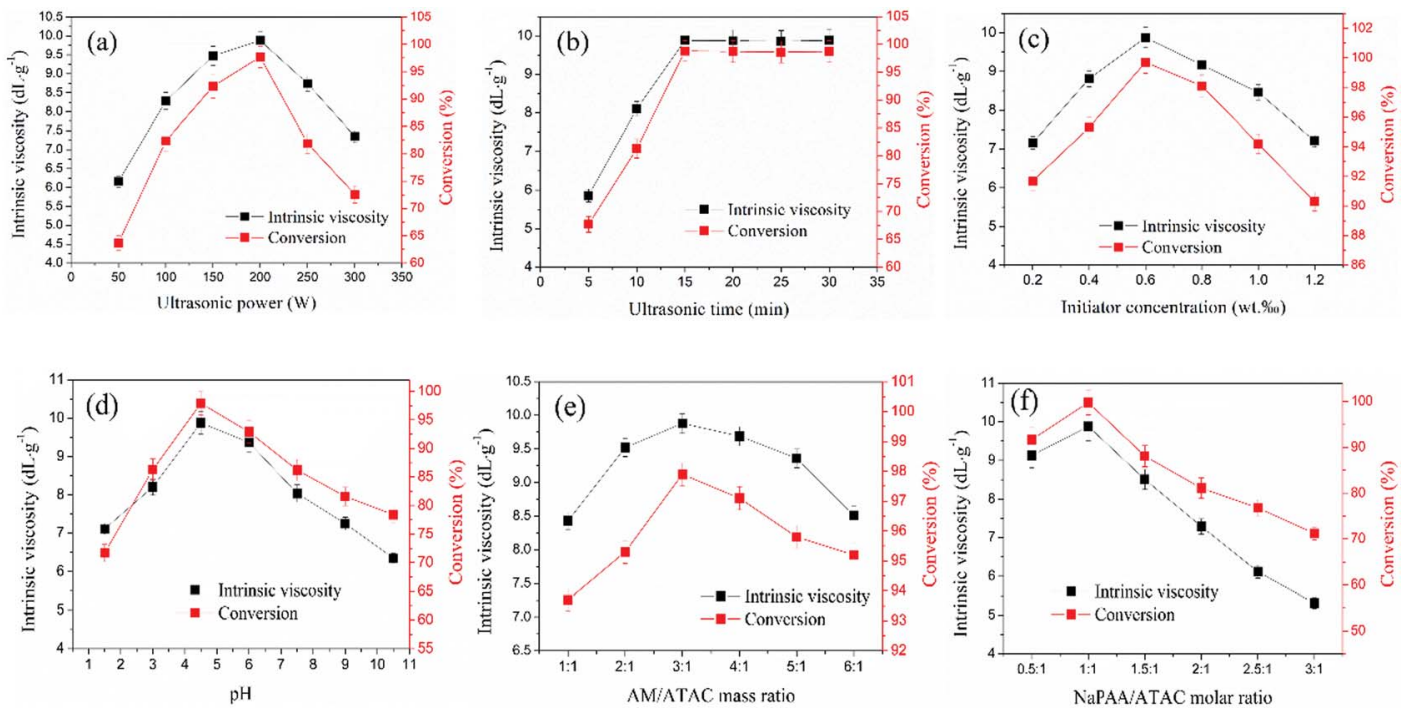

Fig. 3 Effects of (a) ultrasound power, (b) ultrasound time, (c) initiator concentration, (d) pH, (e) AM/ATAC mass ratio and (f) NaPAA/ATAC molar ratio on polymerization.

conversion. However, an excessive initiator dosage would accelerate chain transfer and chain termination and thus cause a decline in intrinsic viscosity and conversion. ${ }^{32}$ In addition, the crosslinking and collapse caused by the excess initiator were unfavorable for increases in intrinsic viscosity and conversion. Thus, the optimal monomer concentration was deemed to be $0.06 \mathrm{wt} \%$ in this study.

3.1.4. Effect of $\mathrm{pH}$ on copolymerization. According to a previous study, $\mathrm{pH}$ is an important factor that significantly affects copolymerization reactions. Other conditions remained constant, and the effect of $\mathrm{pH}$ on the intrinsic viscosity and conversion was investigated. The results shown in Fig. 3(d) indicate that the intrinsic viscosity and conversion greatly increased with an increase in $\mathrm{pH}$ from 1.5 to 4.5 , but a distinct decrease occurred with a further increase in $\mathrm{pH}$. At a lower $\mathrm{pH}$ ( $\mathrm{pH} \leq 3.0)$, intramolecular imidization of AM easily occurred to form a branched or crosslinked polymer, which thereby reduced the intrinsic viscosity and conversion of the copolymer. ${ }^{33}$ Moreover, the strongly acidic conditions caused the acidification of the $-\mathrm{COO}^{-}$group in NaPAA to form a $-\mathrm{COOH}$ group, which reduced the solubility of NaPAA and hindered the copolymerization reaction. In contrast, a high $\mathrm{pH}(\mathrm{pH}>9)$ would accelerate the hydrolysis of the quaternary ammonium group in the ATAC monomer and hinder chain growth, resulting in a higher intrinsic viscosity and conversion..$^{34}$ Therefore, the optimum $\mathrm{pH}$ value for copolymerization was set at 4.5.

3.1.5. Effect of AM/ATAC mass ratio on copolymerization. Fig. 3(e) shows the effect of varying the AM/ATAC mass ratio on the intrinsic viscosity and conversion while the other factors were kept constant. It was obvious that both the intrinsic viscosity and the conversion initially increased to a maximum and then decreased with a further increase in the value of $m_{\mathrm{AM}}: m_{\mathrm{ATAC}}$. When the AM/ATAC mass ratio was $3: 1$, the intrinsic viscosity and conversion reached their maximum values of $9.88 \mathrm{dL} \mathrm{g}^{-1}$ and $99.7 \%$, respectively. In the early stage of copolymerization, the number of free radicals and the chance of collisions between monomers were vital to the intrinsic viscosity and conversion of the polymer. ${ }^{35}$ Because AM has a higher reactivity ratio in comparison with other monomers, a higher AM concentration increased the number of primary free radicals and the chance of collisions, and hence chain propagation and further conversion were more likely to happen. However, an excessive concentration of AM monomer reacted vigorously and thereby accelerated chain transfer and chain termination. As a result, it led to lower intrinsic viscosity and conversion. Therefore, the optimal mass ratio of AM to ATAC was deemed to be $3: 1$.

3.1.6. Effect of NaPAA/ATAC molar ratio on polymerization. Fig. $3(\mathrm{f})$ shows the effect of varying $n_{\mathrm{NaPAA}}: n_{\mathrm{ATAC}}$ on the intrinsic viscosity and conversion while the other factors were constant. It was clear that the intrinsic viscosity and conversion changed greatly at different $n_{\mathrm{NaPAA}}: n_{\mathrm{ATAC}}$ values, which indicated that NaPAA had a great effect on the polymerization. The intrinsic viscosity and conversion initially increased, reached their maximum value at $n_{\mathrm{NaPAA}}: n_{\mathrm{ATAC}}=1: 1$ and then decreased rapidly with a further increase in $n_{\mathrm{NaPAA}}: n_{\mathrm{ATAC}}$. When the anionic polymer NaPAA was added to the reaction system, the cationic monomer ATAC was adsorbed and distributed along the template molecular chain via electrostatic forces. The reaction of the cationic monomers adsorbed on the NaPAA polymer was easily initiated by free radicals with low hindrance, which thus increased the chain propagation and polymer conversion. In contrast, a higher $n_{\mathrm{NaPAA}}: n_{\mathrm{ATAC}}$ value would reduce the intrinsic viscosity and conversion. Because NaPAA with $-\mathrm{COO}^{-}$groups has high steric hindrance, superfluous NaPAA molecules would wrap the NaPAA polymer to create a cage effect, which would distinctly decrease the chance of collisions with free radicals and inhibit the reaction. As a result, the intrinsic viscosity and conversion of the polymer were both reduced. Therefore, the favorable molar ratio of NaPAA to ATAC was determined to be $1: 1$. 


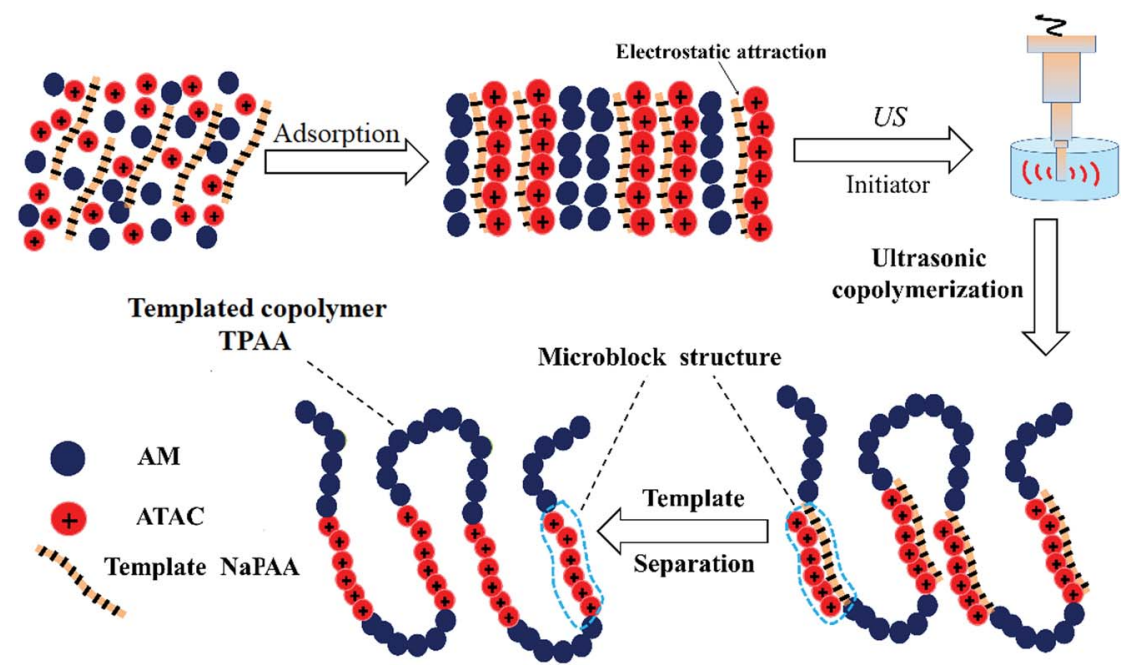

Fig. 4 Possible mechanism of the template copolymerization.

\subsection{Association constant $\left(K_{M}\right)$ and template polymerization mechanism}

In order to obtain an in-depth understanding of the possible template polymerization mechanism, the strength of the interaction between the template and the cationic monomer ATAC was measured via the association constant $\left(K_{\mathrm{M}}\right)$. In this template polymerization system, a $K_{\mathrm{M}}$ value of 13.97 was determined at $n_{\mathrm{NaPAA}}: n_{\mathrm{ATAC}}=1$. The $K_{\mathrm{M}}$ value of 13.97 indicated that more than three-quarters of the ATAC molecules were pre-adsorbed and arranged along the molecular chain of the NaPAA polymer to form a precursor of the microblock structure under the electrostatic force between ATAC and NaPAA. When the reaction of the ATAC microblock structure was initiated by the US radiation, the cationic ATAC microblock structure was successfully synthesized. Finally, the desired templated copolymer TPAA characterized by the novel cationic microblock structure was obtained after the template NaPAA was separated with alcohol. On the basis of the above analyses, a possible zip (type I) mechanism was proposed for this template polymerization, as shown in Fig. 4.

\subsection{Effect of $\boldsymbol{n}_{\mathrm{NaPAA}} / \boldsymbol{n}_{\mathrm{ATAC}}$ on conversion $\left(C_{\mathrm{v}}\right)$ and polymerization rate $\left(R_{\mathrm{p}}\right)$}

In addition to the investigation based on $K_{\mathrm{M}}$, the effect of the template on the conversion $\left(C_{\mathrm{v}}\right)$ and polymerization rate $\left(R_{\mathrm{p}}\right)$ was investigated to further comprehend the template polymerization system. As shown in Fig. 5(a), it was found that the conversion initially increased to a maximum and then declined with an increase in $n_{\mathrm{NaPAA}} / n_{\mathrm{ATAC}}$. When the $n_{\mathrm{NaPAA}} / n_{\mathrm{ATAC}}$ value was 1 , the maximum value of $C_{\mathrm{v}}$ reached $9.51 \%$. Moreover, the variation trend in $R_{\mathrm{p}}$ shown in Fig. 5(b) was similar to that in $C_{\mathrm{v}}$, and the maximum value of $R_{\mathrm{p}}\left(0.105 \mathrm{~mol} \mathrm{~L}^{-1} \mathrm{~min}\right)$ was also observed at $n_{\mathrm{NaPAA}} / n_{\mathrm{ATAC}}=1$. The above phenomenon could be explained as follows. At a lower $n_{\mathrm{NaPAA}} / n_{\mathrm{ATAC}}$ value $(<1)$, an adequate number of ATAC monomers were adsorbed on the molecular chain of the NaPAA template under the electrostatic force, and thus almost no vacant sites were left. As a result, the reaction of ATAC was rapidly initiated and higher values of $C_{\mathrm{v}}$ and $R_{\mathrm{p}}$ were observed. In contrast, at higher $n_{\mathrm{NaPAA}} / n_{\mathrm{ATAC}}$ values $(>1)$, there were vacant sites and gaps in the molecular chain of the NaPAA template because of a lack of sufficient ATAC monomers to fill the vacant sites on NaPAA. In these conditions, the polymerization reaction was inhibited and controlled by the
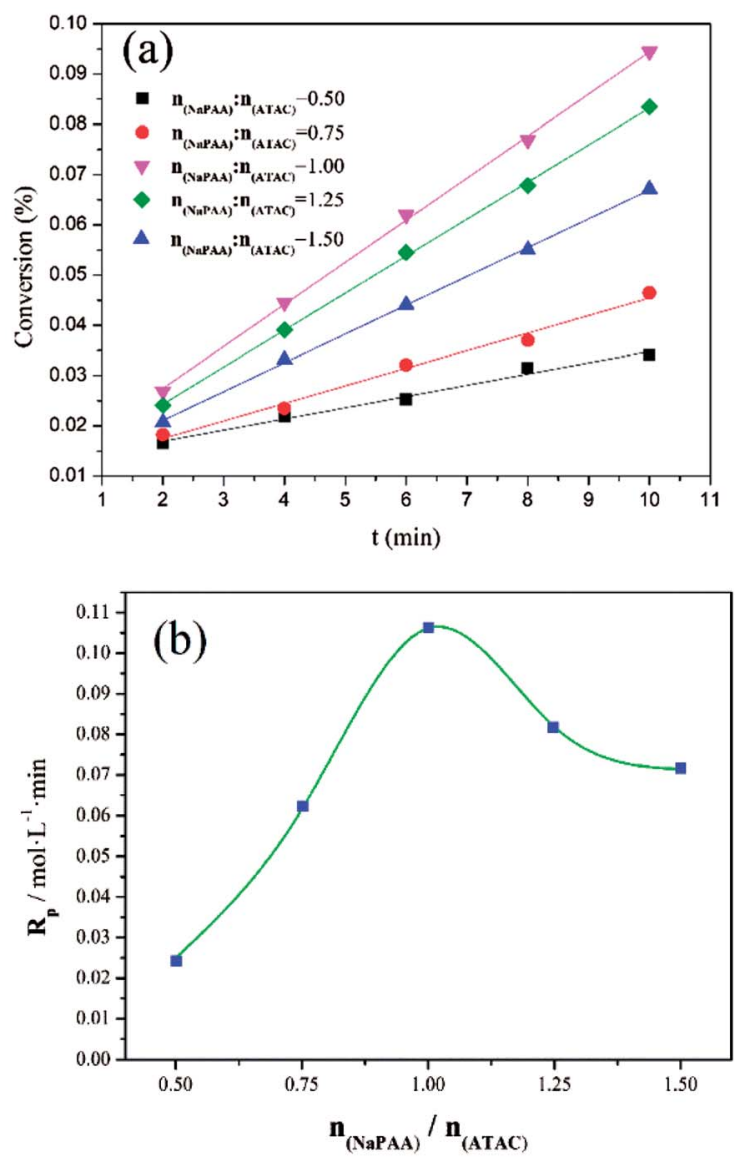

Fig. 5 Effects of $n_{\text {NaPAA }}: n_{\text {ATAC }}$ on (a) conversion $\left(C_{\mathrm{v}}\right)$ and (b) $R_{\mathrm{p}}$ at $m_{\text {AM }}: m_{\text {ATAC }}=3: 1, \mathrm{pH}=4.5$ and $T=30{ }^{\circ} \mathrm{C}$. 
gaps and vacant sites, and therefore a decrease in the variation trends in $C_{\mathrm{v}}$ and $R_{\mathrm{p}}$ was observed. According to the above analyses, the conclusion that a type I (zip) template polymerization mechanism was involved was again confirmed via the investigation based on $C_{\mathrm{v}}$ and $R_{\mathrm{p}}$.

\subsection{Characterization of flocculants}

3.4.1. FTIR spectral analysis. FTIR spectroscopy is regarded as an easy and convenient way to characterize copolymers. ${ }^{36}$ Fig. 6 shows the FTIR spectra of TPAA, CPAA and PAM. The stretching vibrations of $\mathrm{NH}_{2}$ and $\mathrm{C}=\mathrm{O}$ groups in $\mathrm{AM}$ were observed at $3445 \mathrm{~cm}^{-1}$ and $1668 \mathrm{~cm}^{-1}$, respectively. ${ }^{37}$ The stretching vibrations of $\mathrm{CH}_{3}$ groups in $\mathrm{AM}$ appeared at $2940 \mathrm{~cm}^{-1} \cdot{ }^{38}$ Characteristic peaks of TPAA and CPAA appeared at $1543 \mathrm{~cm}^{-1}$ for NH bending vibrations in ATAC, $1494 \mathrm{~cm}^{-1}$ for $\mathrm{CH}_{3}$ stretching vibrations in $-\mathrm{N}^{+}-\left(\mathrm{CH}_{3}\right)_{3}$ groups, $964 \mathrm{~cm}^{-1}$ for $-\mathrm{N}^{+}-\left(\mathrm{CH}_{3}\right)_{3}$ bending vibrations, and $1452 \mathrm{~cm}^{-1}$ for $\mathrm{CH}_{2}$ bending vibrations in $-\mathrm{CH}_{2}-\mathrm{N}^{+}$groups. ${ }^{39}$ In comparison with PAM, the absorption peaks of AM and ATAC all appeared in the FTIR spectra of TPAA and CPAA, which thus indicated the successful copolymerization of AM and ATAC. Moreover, TPAA and CPAA displayed the same absorption peaks, and new absorption peaks were not discovered for either TPAA or CPAA. Therefore, the conclusion could be drawn that US-initiated template polymerization had no influence on the chemical functional groups of the copolymers.

3.4.2. ${ }^{1}$ H NMR spectral analysis. To further confirm the microstructure of the prepared polymer, the ${ }^{1} \mathrm{H}$ NMR spectra of PAM, CPAA and TPAA are shown in Fig. 7. Characteristic absorption peaks of PAM are displayed at $\delta=1.66 \mathrm{ppm}$ for $\mathrm{CH}_{2}$ (a) and $\delta=2.23 \mathrm{ppm}$ for $\mathrm{CH}$ (b), respectively. ${ }^{40}$ Owing to the addition of the monomer ATAC, several characteristic peaks of TPAA and CPAA were observed. In the spectra of TPAA and CPAA, peaks due to ATAC were observed at $\delta=3.27 \mathrm{ppm}\left(\mathrm{H}_{\mathrm{c}}\right)$, $\delta=1.36 \mathrm{ppm}\left(\mathrm{H}_{\mathrm{d}}\right), \delta=3.34 \mathrm{ppm}\left(\mathrm{H}_{\mathrm{e}}\right)$ and $\delta=3.13 \mathrm{ppm}\left(\mathrm{H}_{\mathrm{f}}\right){ }^{31,41}$ Moreover, chemical shifts due to $\mathrm{CH}_{2}$ (a) and $\mathrm{CH}$ (b) were observed in the spectra of TPAA and CPAA. Because of the

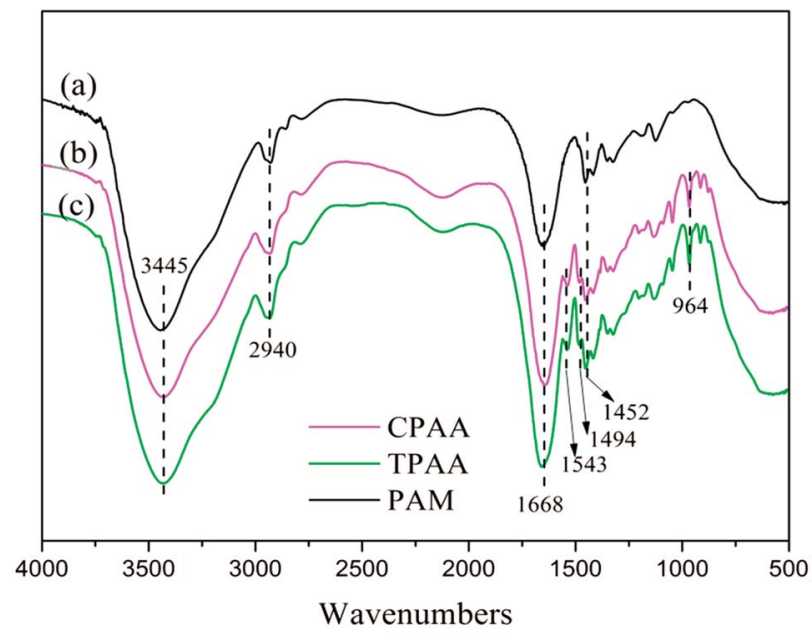

Fig. 6 FTIR spectra of (a) PAM, (b) CPAA and (c) TPAA. grafting of ATAC, the chemical structures of TPAA and CPAA were different from that of PAM, which thus resulted in a chemical shift. The above ${ }^{1} \mathrm{H}$ NMR spectral analyses indicated that TPAA and CPAA were successfully formed by copolymerization of AM and ATAC. However, several subtle differences between TPAA and CPAA should not be ignored and were vital for the formation of the microblock structure. Four additional peaks ( $\delta=2.68 \mathrm{ppm}, \delta=3.03 \mathrm{ppm}, \delta=3.40 \mathrm{ppm}$ and $\delta=3.74$ ppm) marked with triangular symbols appeared in the ${ }^{1} \mathrm{H}$ NMR spectrum of CPAA, whereas these did not appear in the ${ }^{1} \mathrm{H}$ NMR spectrum of TPAA. Besides, the intensity of the peak at $1.99 \mathrm{ppm}$ was high in the spectrum of CPAA but became low in that of TPAA. The difference between TPAA and CPAA was connected to their stereochemical properties. Because of the formation of the cationic microblocks in TPAA, the chemical environments of the protons in the chemical groups $\left(\mathrm{CH}, \mathrm{CH}_{2}-\mathrm{N}^{+}, \mathrm{N}^{+}-\left(\mathrm{CH}_{3}\right)_{3}\right)$ in the TPAA polymer chain became identical, and only one resonance peak was observed for each group in principle. ${ }^{42}$ However, the random distribution of the cationic monomer ATAC in CPAA generated a different chemical environment from that in TPAA. Thus, individual split proton peaks with low intensity were observed for each group in CPAA. This phenomenon was in accordance with the analytical results based on the association constant $\left(K_{\mathrm{M}}\right)$ and TG/DSC analysis, which further confirmed that the cationic microblock structure was successfully synthesized in TPAA.

3.4.3. TG/DSC analysis. Fig. 8 shows the TGA thermographs of the polymers, in which three stages of weight loss were observed. The loss of absorbed moisture in the polymers (CPAA: $10.1 \mathrm{wt} \%$, TPAA: $9.9 \mathrm{wt} \%$ ) occurred within the temperature range of $30-195{ }^{\circ} \mathrm{C} .{ }^{43}$ The thermal decomposition of the imine in the amide groups (CPAA: $18.9 \mathrm{wt} \%$, TPAA: $18.1 \mathrm{wt} \%$ ) occurred between the temperatures of 195 and $350{ }^{\circ} \mathrm{C} .{ }^{44}$ Carbonization of the polymeric backbone and the decomposition of $\mathrm{N}^{+}-\left(\mathrm{CH}_{3}\right)_{3}$ groups (CPAA: $52.5 \mathrm{wt} \%$, TPAA: $53.7 \mathrm{wt} \%$ ) were observed in the range of $350-520{ }^{\circ} \mathrm{C} .{ }^{45}$ Obviously, double hump-like heat absorption peaks $\left(383.6\right.$ and $417.1^{\circ} \mathrm{C}$ ) were observed for TPAA, whereas only one absorption peak $\left(421.1^{\circ} \mathrm{C}\right)$ was observed for CPAA. Because there were two different structural blocks (ATAC and AM blocks) in the TPAA polymer chain, their decomposition generated two different heat absorption peaks. However, the random distribution of ATAA and AM monomers in the CPAA molecular chain severely hindered the formation of the microblock fragments, and hence only a single absorption peak was observed. The results of thermal analysis provided another proof of the formation of the ATAC microblock structure in the TPAA polymer chain.

3.4.4. SEM of polymers. SEM images of PAM, CPAA and TPAA were obtained, as displayed in Fig. 9, to investigate their amorphous morphology and intuitively observe visualized surface information for the polymers. It was evident that the polymers displayed different surface morphologies. As shown in Fig. 9(a), PAM had a smooth and regular surface morphology similar to that in a previous study ${ }^{46}$ After the grafting of DMC on AM, CPAA and TPAA had rough and coarse surface morphologies, which were significantly different from that of PAM. The reason for this change was that the introduction of 


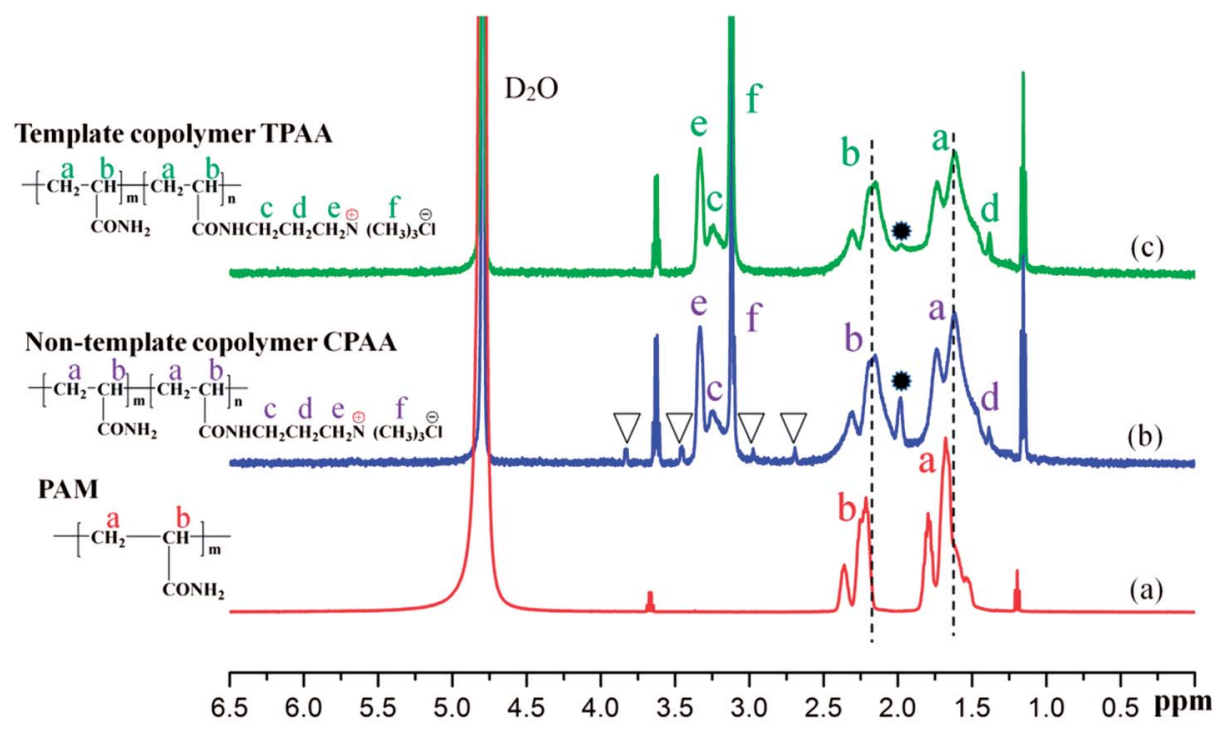

Fig. $7{ }^{1} \mathrm{H}$ NMR spectra of (a) PAM, (b) CPAA and (c) TPAA.

ATAC into the AM chain destroyed the original structure of PAM and thus resulted in a porous and rough surface morphology with an irregular structure. Moreover, CPAA displayed a multilayered folded structure with a less porous surface, whereas TPAA had an evident macroporous structure with a rough and coarse surface after the US-initiated template copolymerization. The difference in surface morphology between CPAA and TPAA was caused by the formation of the cationic microblock structure and the use of US-initiated template copolymerization. The template tightly bound the cationic monomers and occupied a certain spatial location in the initial phase of formation of the cationic microblock structure. Then, with the removal of the template, a certain pore formed at the original position of the template, whereby the porosity of TPAA was significantly increased. Thus, a more ragged and macroporous surface morphology was observed for TPAA. In addition, US-initiated copolymerization has been demonstrated to be a useful method for modifying and increasing the surface porosity of prepared polymers. ${ }^{22}$ According to the results of previous studies, TPAA with a porous structure would have a huge surface area, which could enhance its water solubility and improve its flocculation performance. ${ }^{35,39}$

\section{Flocculation performance}

\subsection{Effect of flocculant dosage}

In a flocculation process, the flocculant dosage and $\mathrm{pH}$ of the wastewater are the two usual main factors used to evaluate the flocculation performance of the flocculants. ${ }^{47}$ In this study, the flocculation performance of CPAA and TPAA, as well as that of commercial flocculants (CCPDA and CCPMA), was examined and compared in terms of residual turbidity and floc fractal dimension $\left(D_{\mathrm{f}}\right)$ at various dosages and $\mathrm{pH}$ values. As shown in Fig. 10, the residual turbidity exhibited a similar variation trend of initially decreasing and then rapidly increasing with an increase in the polymer dosage from $0.5 \mathrm{mg} \mathrm{L}^{-1}$ to $3.5 \mathrm{mg} \mathrm{L}^{-1}$, but the behavior of the $D_{\mathrm{f}}$ value was exactly the opposite. The minimum residual turbidity and maximum $D_{\mathrm{f}}$ value, namely,
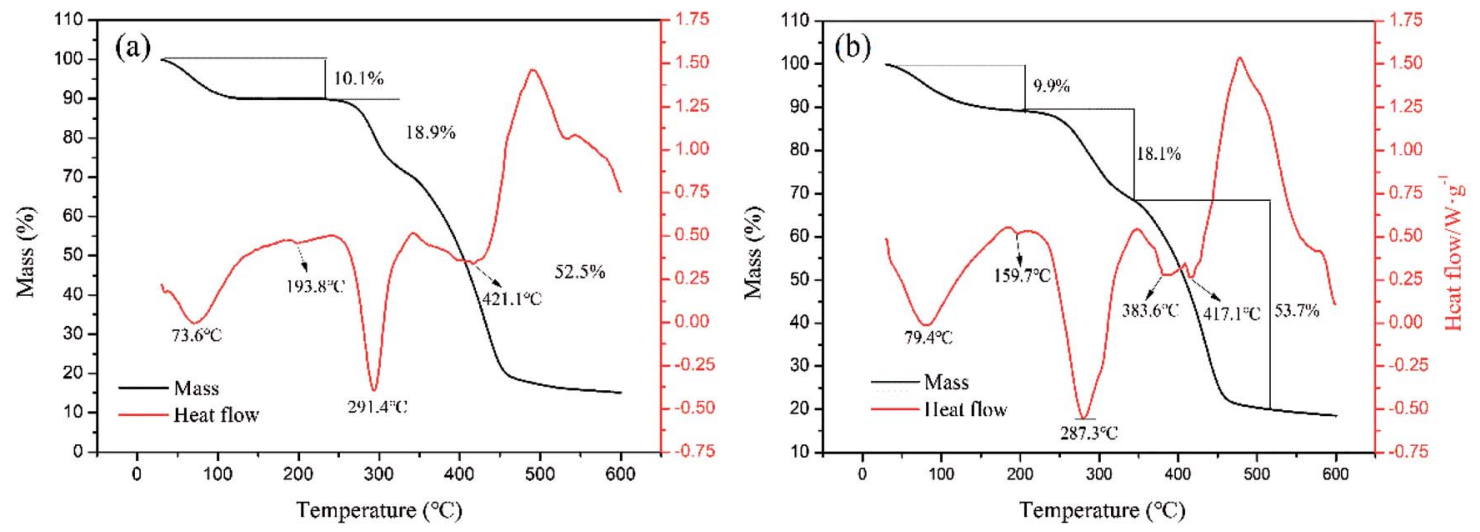

Fig. 8 Thermogravimetric curves of (a) CPAA and (b) TPAA. 

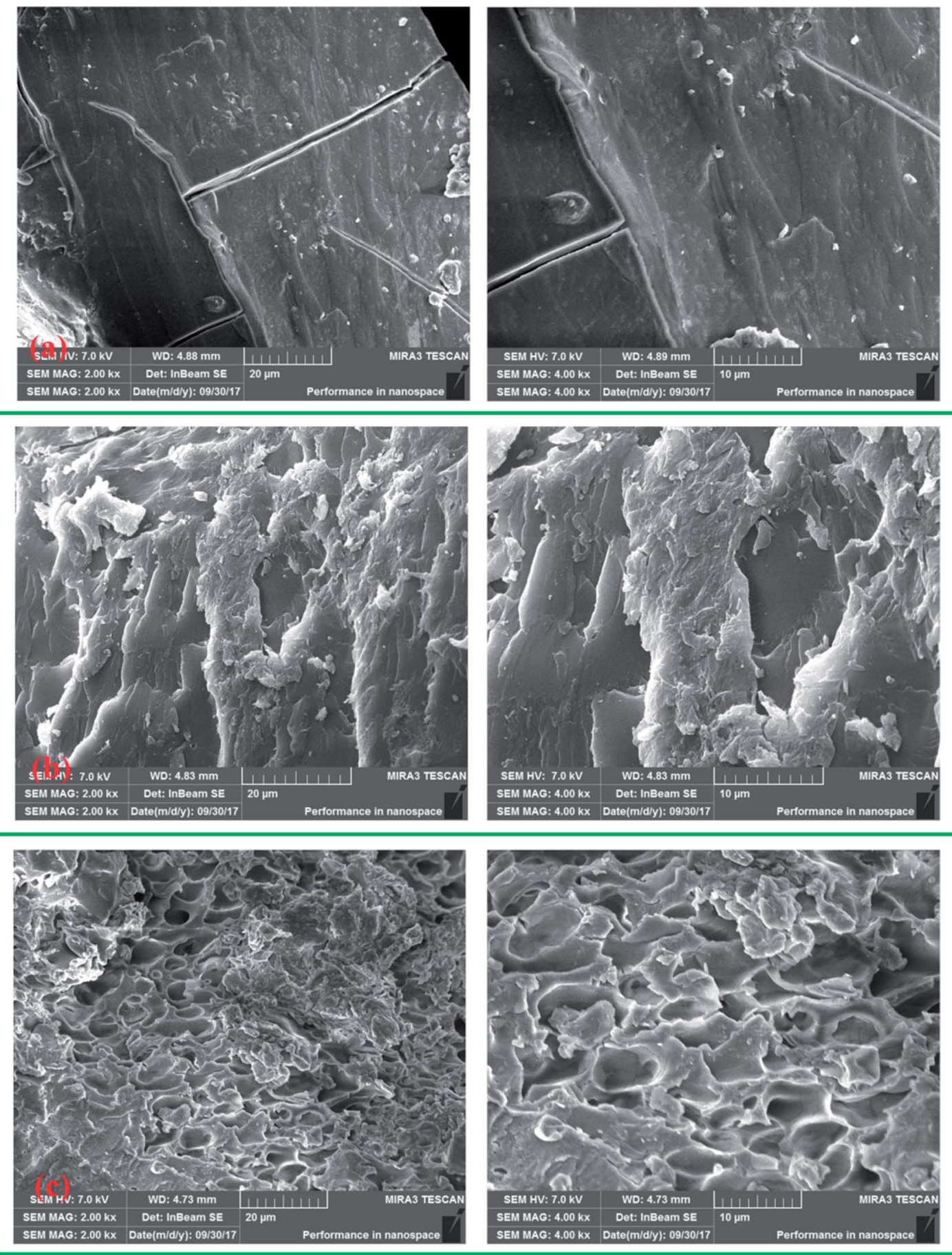

Fig. 9 SEM micrographs of (a) PAM, (b) CPAA and (c) TPAA

5.8 NTU and 1.89, 8.9 NTU and 1.77, 13.9 NTU and 1.72, and 15.8 NTU and 1.64, were observed for TPAA, CPAA, CCPDA and CCPMA, respectively, at a dosage of $2.0 \mathrm{mg} \mathrm{L}^{-1}$. This phenomenon could be explained by the following reasons. Numerous negatively charged kaolin particles would generate strong charge repulsion, which caused the kaolin wastewater system to become more stable and thus led to higher turbidity. At low flocculant dosages, insufficient positive charges were available to neutralize the kaolin colloids, as well as inadequate surface adhesion to intercept suspended kaolin particles. ${ }^{48}$ Consequently, a sufficient dose of the cationic flocculant was urgently required to neutralize the negatively charged kaolin particles and adsorb them. However, with an excessive flocculant dosage, suspended kaolin particles were surrounded by cationic polymers, which resulted in a caking effect, and the electrostatic repulsion between fine aggregates also increased. ${ }^{49}$ As a result, the fine aggregates became more unstable and decomposed to form a fluffy and uncompacted floc structure with a low $D_{\mathrm{f}}$ 

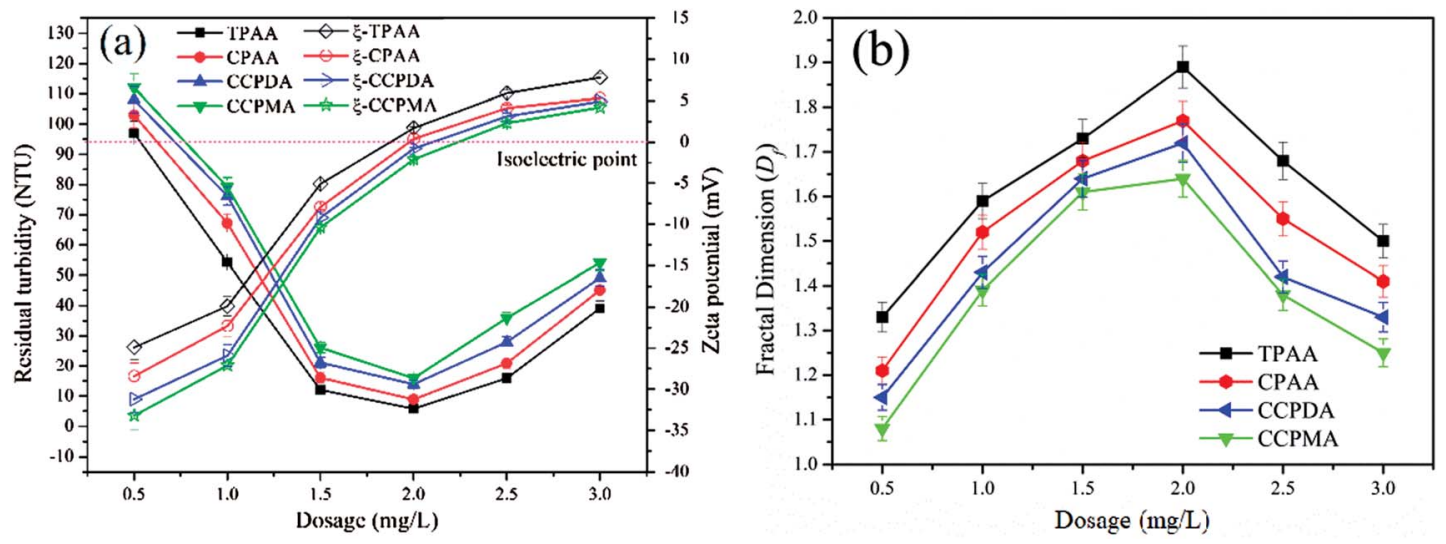

Fig. 10 Effect of dosage on (a) residual turbidity and zeta potential and (b) floc fractal dimension $\left(D_{\mathrm{f}}\right)$.

value, as shown in Fig. 10(b). Moreover, TPAA displayed superior flocculation performance, in terms of lower residual turbidity and a higher $D_{\mathrm{f}}$ value, than the other three flocculants (CPAA, CCPDA and CCPMA) over the entire dosage range. The excellent flocculation performance of TPAA was attributed to the cationic microblocks. Owing to the formation of cationic microblocks in the polymer chain of TPAA, its charge neutralization and patching abilities were greatly enhanced (TPAA had the highest zeta potential value among the flocculants listed in Fig. 10(a)). Therefore, increasing numbers of negatively charged kaolin particles were neutralized and thoroughly destabilized by the cationic microblocks and thus formed large and compact flocs. In addition, the cationic microblocks in TPAA generated a ragged and macroporous surface morphology, which was helpful for the rapid dissolution of the polymer chain to adsorb and capture more kaolin particles. Consequently, desirable flocculation performance with low residual turbidity and a denser floc structure was observed for TPAA.

\subsection{Effect of $\mathbf{p H}$}

The effect of $\mathrm{pH}$ on the flocculation performance was evaluated at a dosage of $2.0 \mathrm{mg} \mathrm{L}{ }^{-1}$, and the results are displayed in Fig. 11. Obviously, different flocculation performance was observed for each flocculant, which indicated that the $\mathrm{pH}$ exerted a significant influence on the removal of kaolin particles. At a lower $\mathrm{pH}$, numerous $\mathrm{H}^{+}$ions generated by the strongly acidic environment would cause the protonation of amino groups $\left(\mathrm{NH}_{3}^{+}\right)$, as well as strong charge repulsion between kaolin particles, and thus lessen the charge neutralization and adsorption bridging ability. ${ }^{50}$ Consequently, a deterioration in flocculation performance, with higher residual turbidity and a lower $D_{\mathrm{f}}$ value, occurred. Conversely, a higher $\mathrm{pH}$ increased the concentration of charged hydroxide ions $\left(\mathrm{OH}^{-}\right)$and caused the deprotonation of $\mathrm{NH}_{2}$ groups, which also had adverse effects with respect to the enhancement of the charge neutralization and adsorption bridging ability of the flocculants. ${ }^{51}$ After the addition of the flocculants, the zeta potential of the supernatant significantly increased, which indicated that charge neutralization played an important role in the flocculation process. Moreover, it was found that TPAA exhibited the most prominent flocculation performance (the lowest residual turbidity of 5.8 NTU, and the highest $D_{\mathrm{f}}$ value of 1.89) among the flocculants over the entire $\mathrm{pH}$ range. Owing to the cationic microblocks in the TPAA molecular chain, the charge neutralization and patching ability was greatly increased, whereby the zeta potential of the supernatant treated with TPAA was the highest among the four flocculants. During the flocculation process, more negatively charged kaolin particles were neutralized completely for the next steps of adsorption and bridging. Besides, the cationic microblocks would result in strong repulsion, which was more favorable for the stretching and extension of the polymer chain and thus improved the bridging effect. ${ }^{52}$ In these conditions, kaolin particles were captured and anchored by the molecular chain of TPAA to form a large and compact floc structure. Finally, TPAA was suitable over a wider $\mathrm{pH}$ range from 5.0 to 9.0, in which TPAA displayed higher flocculation efficiency for the removal of turbidity due to kaolin.

\subsection{Effect of flocculants on settling behavior of kaolin}

As previous studies revealed, aspects of the settling behavior of kaolin such as the floc size and floc kinetic can be used as acceptable indices to evaluate the flocculation performance and efficiency of flocculants. ${ }^{53,54}$ In general, it was suggested that a higher floc kinetic and a large floc size corresponded to excellent flocculation ability. ${ }^{55}$ Flocs with a small size and a low floc kinetic would become more mobile and randomly distributed in wastewater, which would thus increase the difficulty of separation and reduce the flocculation efficiency. Therefore, it was significant to investigate the floc kinetic and floc size after flocculation under the optimal flocculation conditions (dosage of $2.0 \mathrm{mg} \mathrm{L}^{-1}$ and $\mathrm{pH}$ of 7.0), and the results are illustrated in Fig. 12 and 13.

As shown in Fig. 12(a), similar variations in the residual turbidity of kaolin, which rapidly decreased at first and then slowly declined to a minimum value, were observed for the four flocculants. However, the turbidity of kaolin treated with TPAA decreased sharply during the first $7 \mathrm{~min}$, which was associated with the largest slope $\left(k N_{0}=15.86 \times 10^{-4} \mathrm{~s}^{-1}\right)$ among the flocculants. The above phenomenon suggested that the cationic 

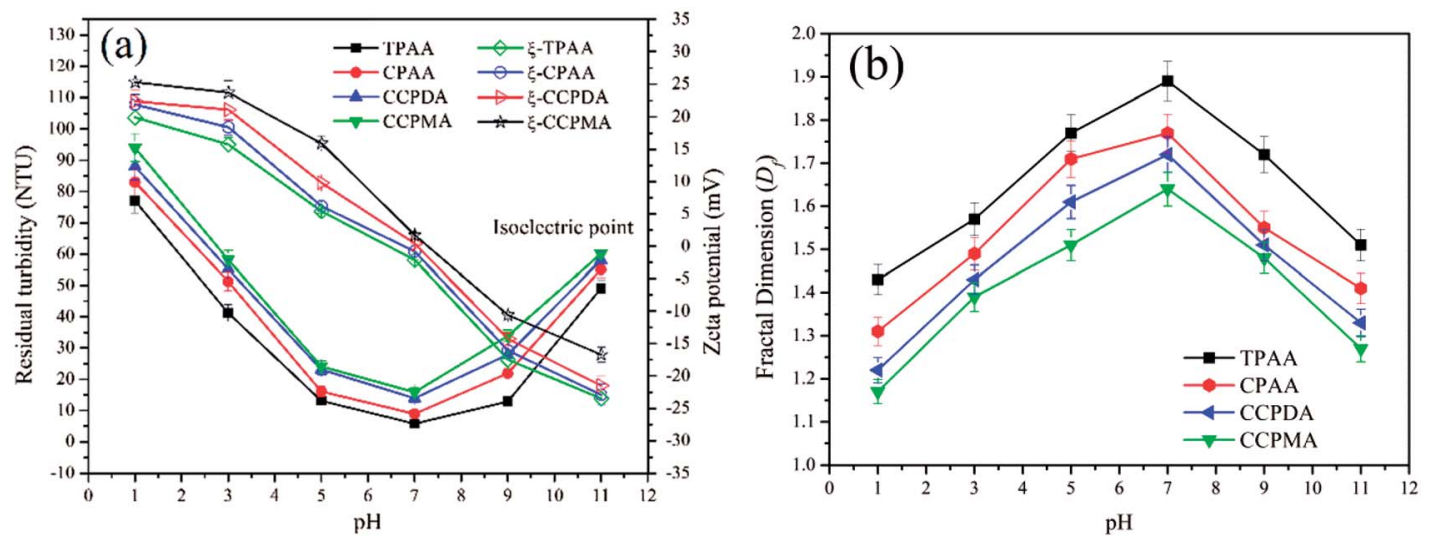

Fig. 11 Effect of $\mathrm{pH}$ on (a) residual turbidity and zeta potential and (b) floc fractal dimension $\left(D_{\mathrm{f}}\right)$.

microblock structure in TPAA could greatly improve the flocculation performance. The strong charge neutralization and patching ability generated by the cationic microblocks could attract and thoroughly neutralize the negatively charged kaolin particles and thereby cause kaolin colloids to lose their stability for the next flocculation step of adsorption and bridging. ${ }^{56}$ Besides, the cationic microblocks in TPAA would lead to strong electrostatic repulsion, which is favorable for the stretching and extension of polymer chains. ${ }^{52}$ In this case, numerous kaolin particles would be intercepted efficiently and anchored on the polymer chain of TPAA to aggregate and form a large and compact floc structure. Therefore, the residual turbidity of the kaolin wastewater conditioned by TPAA decreased rapidly, and desirable flocculation performance was achieved (Table 2).

Fig. 13 illustrates the size distribution after flocculation of kaolin flocs formed by TPAA, CPAA, CCPDA and CCPMA. The floc size $d_{50}$ (median equivalent volumetric diameter) was selected as the evaluation index, and the results showed that the $d_{50}$ values for TPAA, CPAA, CCPDA and CCPMA were 608.404, $563.685,490.760$, and $446.802 \mu \mathrm{m}$, respectively. Clearly, TPAA gave rise to a larger floc size than the other three flocculants, which indicated that TPAA was superior in the separation and removal of kaolin. The large floc size was ascribed to the cationic microblocks in TPAA. On the one hand, the cationic microblocks could lead to strong charge neutralization and patching ability, and hence TPAA could neutralize more negatively charged kaolin particles to form large and compact flocs. On the other hand, the strong electrostatic repulsion generated by the cationic microblocks was favorable for the extension of the molecular chain. Thus, the bridging ability of TPAA was greatly enhanced, and a large kaolin floc size was attained.

\subsection{FTIR analysis of the formed kaolin flocs}

In order to analyze the interaction between the flocculants and kaolin particles and further understand the mechanism involved in the flocculation process, the FTIR spectra of flocs generated by kaolin + TPAA and kaolin + CPAA were compared. As shown in Fig. 14, the characteristic absorption peaks of kaolin particles were observed at $1118 \mathrm{~cm}^{-1}$ and $556 \mathrm{~cm}^{-1}$, which were ascribed to vibrations of $\mathrm{Si}-\mathrm{O}$ and $\mathrm{Si}-\mathrm{O}-\mathrm{Al}$ groups, respectively ${ }^{57,58}$ After flocculation, these absorption peaks both appeared in the FTIR spectra of kaolin + TPAA and kaolin + CPAA. Moreover, the characteristic absorption peaks of TPAA and CPAA were all observed in the FTIR spectra of kaolin + TPAA and kaolin + CPAA. For example, the peaks at $2920 \mathrm{~cm}^{-1}$,
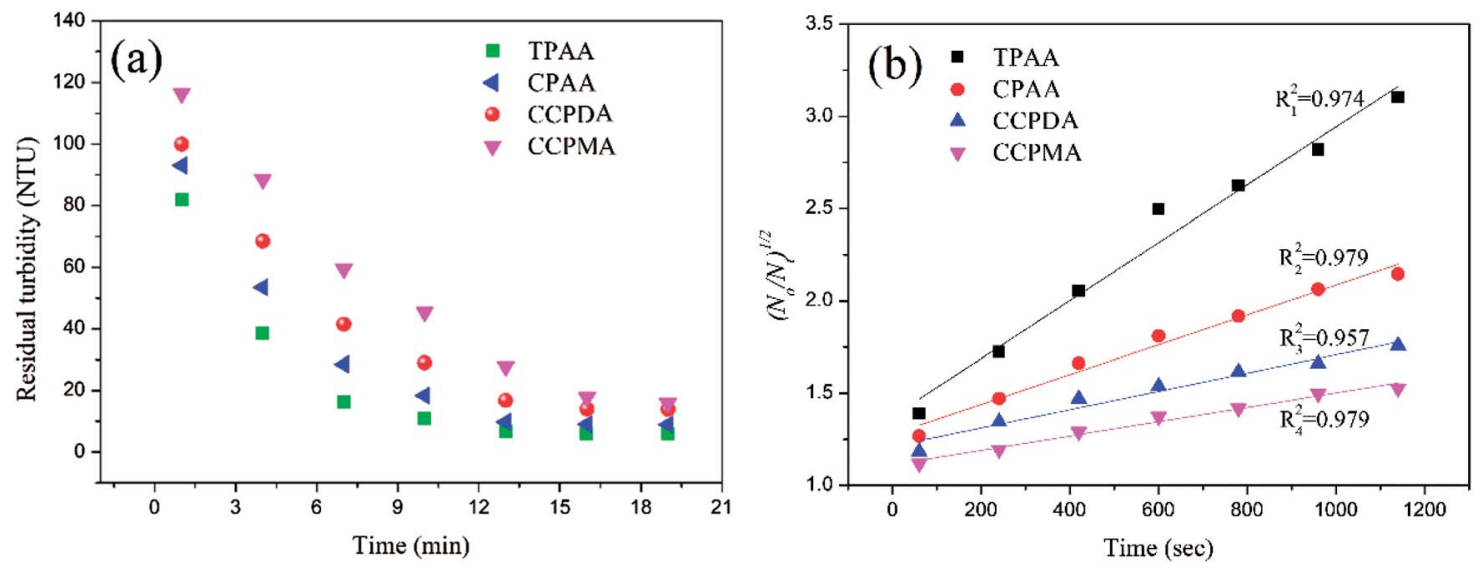

Fig. 12 (a) Variations in residual turbidity and (b) $\left(N_{0} / N_{t}\right)^{1 / 2}$ as a function of settling time for different flocculants. 


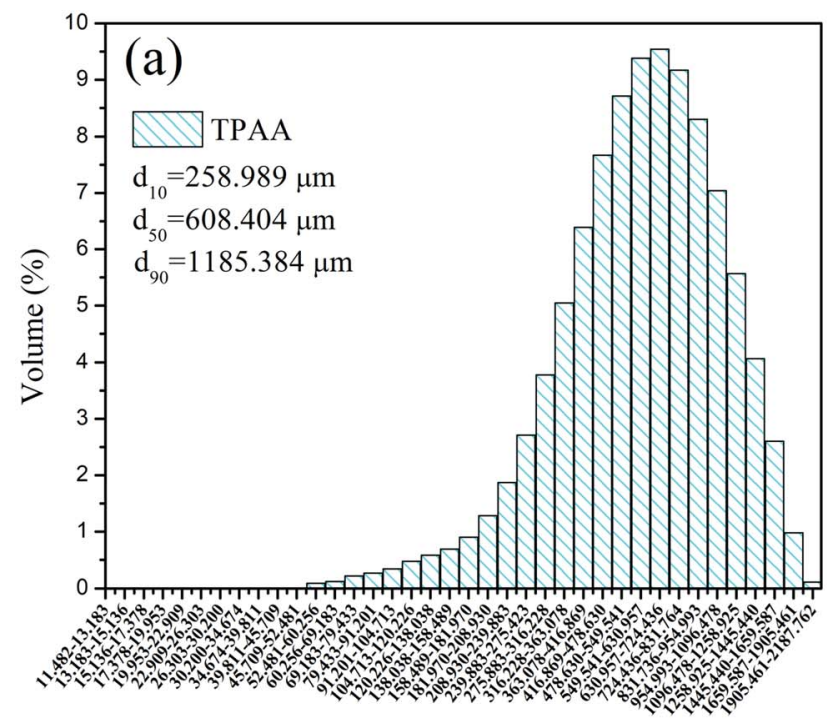

Floc size $(\mu \mathrm{m})$

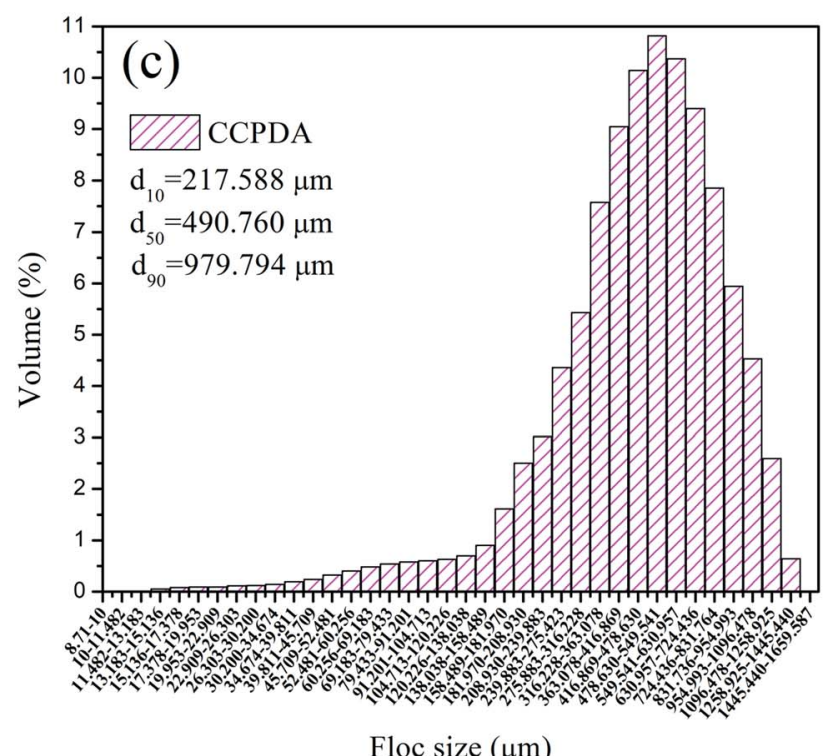

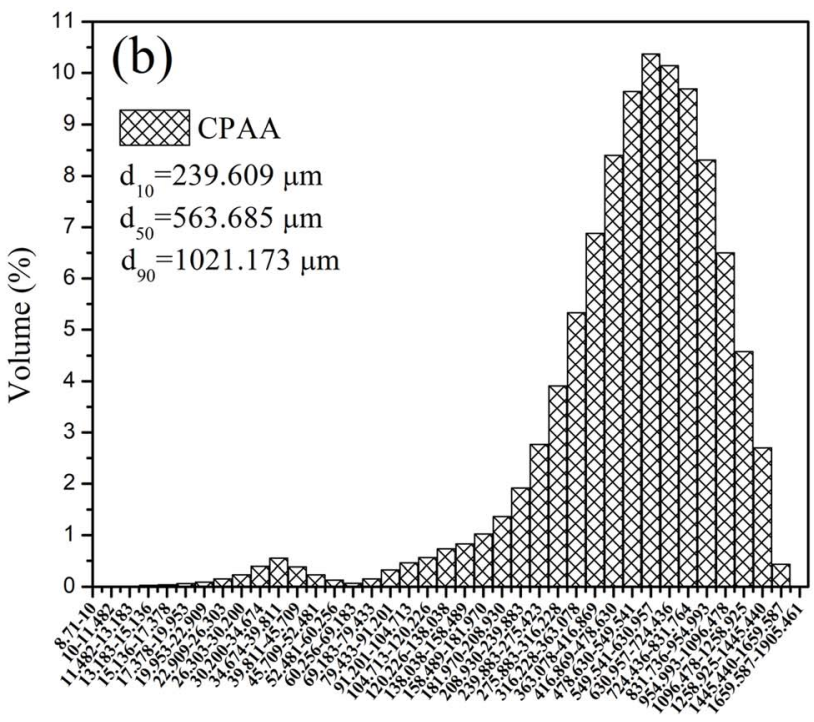

Floc size $(\mu \mathrm{m})$

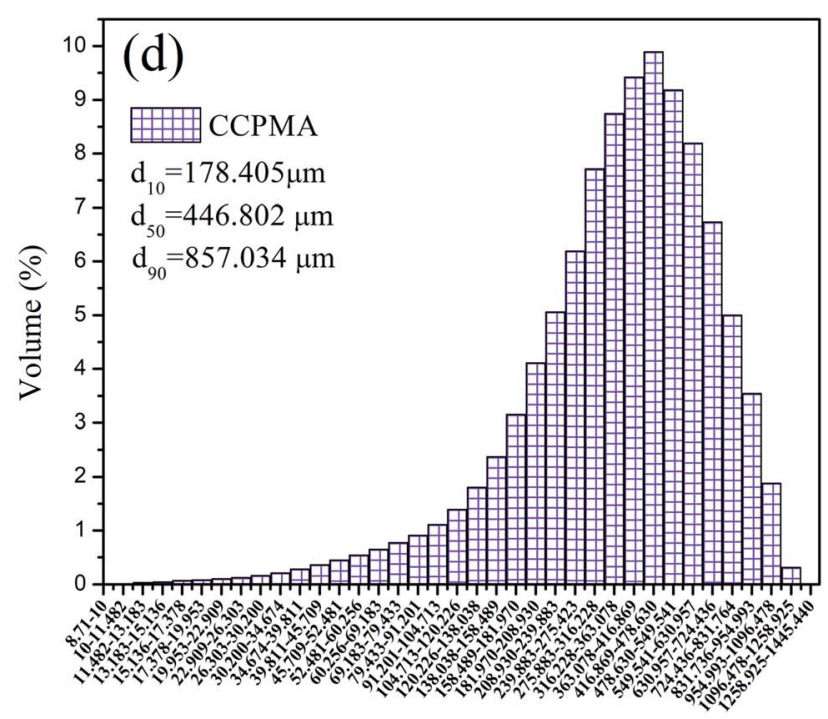

Floc size $(\mu \mathrm{m})$

Fig. 13 Size distribution of kaolin floc formed by (a) TPAA, (b) CPAA, (c) CCPDA and (d) CCPMA.

Table 2 Flocculation kinetics $\left(k N_{0}\right)$ for the four flocculants (TPAA, CPAA, CCPDA and CCPMA)

\begin{tabular}{lcc}
\hline & Flocculation kinetics & \\
Flocculant & $k N_{0}\left(\times 10^{-4} \mathrm{~s}^{-1}\right)$ & $R^{2}$ \\
\hline TPAA & 15.86 & 0.974 \\
CPAA & 8.21 & 0.979 \\
CCPDA & 5.24 & 0.957 \\
CCPMA & 3.78 & 0.979
\end{tabular}

$1661 \mathrm{~cm}^{-1}, 1543 \mathrm{~cm}^{-1}$ and $964 \mathrm{~cm}^{-1}$ were assigned to $\mathrm{CH}_{3}, \mathrm{C}=$ $\mathrm{O}, \mathrm{NH}$ and $\mathrm{N}^{+}-\left(\mathrm{CH}_{3}\right)_{3}$ groups, respectively. The above results indicated the successful occurrence of flocculation between kaolin particles and the flocculants, as well as the removal of kaolin particles from water. However, new chemical bonds were not observed in a comparison of the FTIR spectra of kaolin, kaolin + TPAA and kaolin + CPAA, which indicated that a chemical reaction did not occur between kaolin and the flocculants, and the flocs were mainly formed by electrostatic interaction, patching and bridging.

\section{Flocculation mechanism}

On the basis of the investigation of the zeta potential, floc size and fractal dimension, and floc kinetic and the evaluation of the flocculation performance for kaolin, a possible flocculation mechanism for the templated polymer TPAA was proposed, as shown in Fig. 15. It is more acceptable and straightforward to understand the flocculation mechanism from the following 


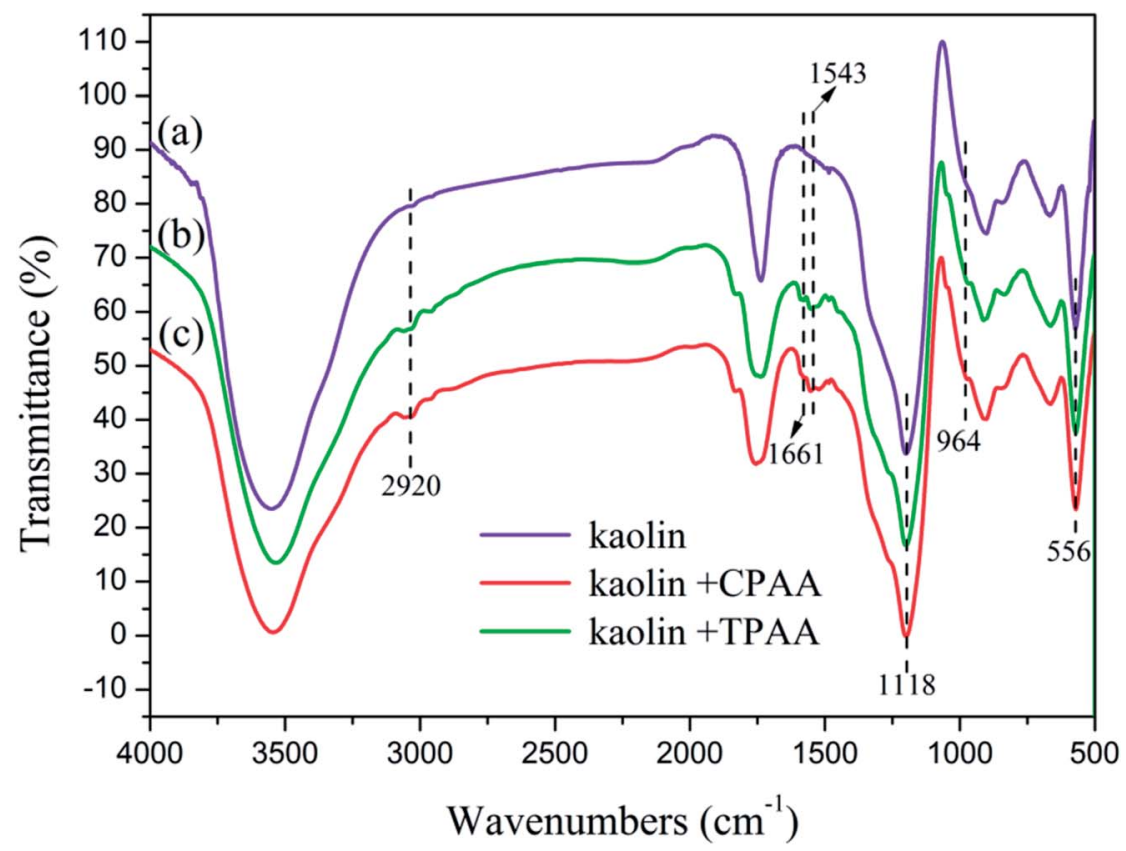

Fig. 14 FTIR spectra of kaolin particles and flocs.

aspects: (1) the charge neutralization and patching ability of TPAA was greatly enhanced owing to the formation of cationic microblocks in TPAA. Thus, negatively charged kaolin particles were neutralized completely and hence lost their colloidal stability. Besides, electrostatic patching was also more beneficial for the occurrence of the desired flocculation, because the adsorption of a polymer with a cationic microblock structure onto local sites of particles caused patches of local charge reversal and resulted in positive-negative attraction between the particles. (2) The cationic microblocks were helpful for improving the bridging effect, because the strong charge repulsion generated by the cationic microblocks was favorable for stretching and extension of the polymer chain. (3) The FTIR analysis of the kaolin flocs that formed confirmed that charge neutralization and bridging were dominant in the kaolin flocculation process. (4) Owing to the enhanced charge neutralization and bridging effect of the flocculant TPAA with its cationic microblock structure, numerous negatively charged kaolin particles were neutralized and adsorbed to aggregate and form large and compact flocs. These large and compact flocs contributed greatly to the achievement of a rapid settling rate and desirable flocculation performance in the removal and separation of kaolin, which thus decreased the turbidity due to kaolin.

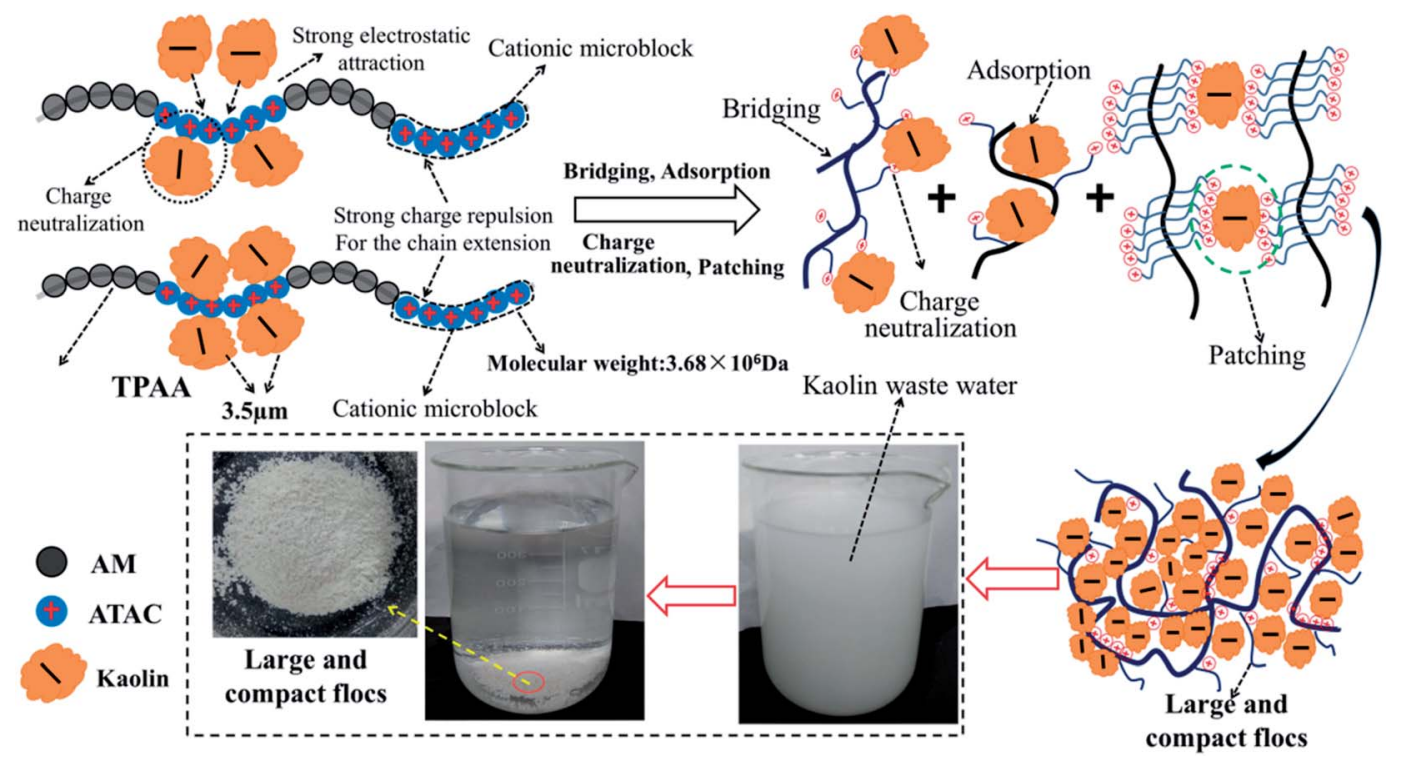

Fig. 15 Possible flocculation mechanism of TPAA. 


\section{Conclusions}

A new cationic flocculant, namely, TPAA, with a cationic microblock structure was successfully prepared for the treatment of high-turbidity wastewater. US-initiated template polymerization was employed to synthesize the templated copolymer TPAA using AM and ATAC as the monomers and NaPAA as the template. The conditions that affected the copolymerization to form TPAA were investigated, and the optimized conditions were an ultrasound power of $200 \mathrm{~W}$, an ultrasound time of $15 \mathrm{~min}$, an initiator concentration of $0.06 \mathrm{wt} \%$, a $\mathrm{pH}$ of 4.5 , an $m_{\mathrm{AM}}: m_{\mathrm{ATAC}}$ ratio of $3: 1$ and an $n_{\mathrm{NaPAA}}: n_{\mathrm{ATAC}}$ ratio of 1 . Under these conditions, an intrinsic viscosity of $9.88 \mathrm{dL} \mathrm{g}^{-1}$ and a conversion of $99.7 \%$ were achieved using TPAA. The structural properties of TPAA were characterized by analytical techniques such as FTIR, ${ }^{1} \mathrm{H}$ NMR, TGA and SEM, and the results indicated the successful formation of the cationic microblock structure. Moreover, a type I (zip) template polymerization mechanism was determined from the results of analyses based on $K_{\mathrm{M}}$ (13.97), $C_{\mathrm{v}}(9.51 \%)$ and $R_{\mathrm{p}}\left(0.105 \mathrm{~mol} \mathrm{~L}^{-1} \mathrm{~min}\right)$, which also provided another convincing proof of the generation of the cationic microblock structure. A flocculation test involving the treatment of high-turbidity water confirmed that the microblock structure in TPAA could significantly increase its charge neutralization, patching and bridging ability, which contributed greatly to excellent flocculation performance (turbidity: 5.8 NTU, $D_{\mathrm{f}}: 1.89$, floc size $d_{50}: 608.404 \mu \mathrm{m}$ and floc kinetic: $15.86 \times$ $10^{-4} \mathrm{~s}^{-1}$ ). FTIR analysis of the kaolin flocs that formed indicated that the cationic microblock structure could generate a strong and powerful bond between TPAA and kaolin particles, but not chemical bonds, via the effect of charge neutralization, patching and bridging. In this case, the flocs generated by TPAA were dense and large, which thus resulted in a rapid settling rate for the removal of kaolin particles and the reduction of their high turbidity.

\section{Conflicts of interest}

There are no conflicts to declare.

\section{Acknowledgements}

The authors are grateful for the financial support provided by the National Natural Science Foundation of China (Project No. 21477010) and China Postdoctoral Science Foundation funded project (Project No. 2017M622970).

\section{References}

1 J. Ma, J. Shi, H. Ding, G. Zhu, K. Fu and X. Fu, Chem. Eng. J., 2017, 312, 20-29.

2 A. L. Mather and R. L. Johnson, J. Hydrol., 2015, 530, 751761.

3 R. Das, S. Ghorai and S. Pal, Chem. Eng. J., 2013, 229, 144152.

4 C. Hu, S. Lo, C. Chang, F. Chen, Y. Wu and J. Ma, Sep. Purif. Technol., 2013, 104, 322-326.
5 G. Muthuraman and S. Sasikala, J. Ind. Eng. Chem., 2014, 20, 1727-1731.

6 M. Huang, Y. Wang, J. Cai, J. Bai, H. Yang and A. Li, Water Res., 2016, 98, 128-137.

7 W. Ren, A. Zhang, S. Qin and Z. Li, Carbohydr. Polym., 2016, 144, 238-244.

8 X. Huang, B. Gao, Y. Sun, Q. Yue, Y. Wang, Q. Li and X. Xu, Sep. Purif. Technol., 2017, 173, 209-217.

9 B. Xu, C. Zheng, H. Zheng, Y. Wang, C. Zhao, C. Zhao and S. Zhang, RSC Adv., 2017, 7, 47029-47037.

10 A. Ariffin, R. S. A. Shatat, A. R. N. Norulaini and A. K. M. Omar, Desalination, 2005, 173, 201-208.

11 T. Nasim and A. Bandyopadhyay, Sep. Purif. Technol., 2012, 88, 87-94.

12 J. Ma, K. Fu, L. Jiang, L. Ding, Q. Guan, S. Zhang, H. Zhang, J. Shi and X. Fu, Sep. Purif. Technol., 2017, 181, 201-212.

13 F. Yang, X. Wang, M. Li, X. Liu, X. Zhao, D. Zhang, Y. Zhang, J. Yang and Y. Li, Acc. Chem. Res., 2016, 49, 606-615.

14 M. Guli, E. M. Lambert, M. Li and S. Mann, Angew. Chem., 2010, 49, 520-523.

15 X. Xiao, H. Song, S. Lin, Y. Zhou, X. Zhan, Z. Hu, Q. Zhang, J. Sun, B. Yang, T. Li, L. Jiao, J. Zhou, J. Tang and Y. Gogotsi, Nat. Commun., 2016, 7, 11296.

16 Y. Wang, A. S. Angelatos and F. Caruso, Chem. Mater., 2008, 20, 848-858.

17 S. Połowiński, Prog. Polym. Sci., 2002, 27, 537-577.

18 B. Bolto and J. Gregory, Water Res., 2007, 41, 2301-2324.

19 M. Constantin, I. Mihalcea, I. Oanea, V. Harabagiu and G. Fundueanu, Carbohydr. Polym., 2011, 84, 926-932.

20 J. Li, Y. Li, S. Niu and N. Li, Ultrason. Sonochem., 2017, 36, 277-285.

21 B. A. Bhanvase, D. V. Pinjari, S. H. Sonawane, P. R. Gogate and A. B. Pandit, Ultrason. Sonochem., 2012, 19, 97-103.

22 J. Li, Y. Li, Y. Song, S. Niu and N. Li, Ultrason. Sonochem., 2017, 39, 853-862.

23 H. Shang, J. Liu, Y. Zheng and L. Wang, J. Appl. Polym. Sci., 2010, 111, 1594-1599.

24 H. Ren, W. Chen, Y. Zheng and Z. Luan, React. Funct. Polym., 2007, 67, 601-608.

25 X. Wu, W. R. Carroll and K. D. Shimizu, Chem. Mater., 2008, 20, 4335-4346.

26 Z. Hua, S. Zhou and M. Zhao, Biosens. Bioelectron., 2009, 25, 615-622.

27 Y. X. Zhao, S. Phuntsho, B. Y. Gao, X. Huang, Q. B. Qi, Q. Y. Yue, Y. Wang, J. H. Kim and H. K. Shon, Environ. Sci. Technol., 2013, 47, 12966-12975.

28 P. Jarvis, B. Jefferson and S. A. Parsons, Environ. Sci. Technol., 2005, 39, 2307-2314.

29 Y. Fang, X. Liu, X. Liang, H. Liu, X. Gao and Z. Zhang, Appl. Energy, 2014, 132, 551-556.

30 S. K. Ooi and S. Biggs, Ultrason. Sonochem., 2000, 7, 125-133. 31 Y. Liao, H. Zheng, L. Qian, Y. Sun, L. Dai and W. Xue, Ind. Eng. Chem. Res., 2014, 53, 11193-11203.

32 S. A. Seabrook and R. G. Gilbert, Polymer, 2007, 48, 47334741.

33 K. B. Thapa, Y. Qi, S. A. Clayton and A. F. A. Hoadley, Water Res., 2009, 43, 623-634. 
34 Y. Bao, J. Ma and N. Li, Carbohydr. Polym., 2011, 84, 76-82. 35 Y. Sun, C. Zhu, W. Sun, Y. Xu, X. Xiao, H. Zheng, H. Wu and C. Liu, Carbohydr. Polym., 2017, 164, 222-232.

36 T. Chen, Q. Fang, Q. Zhong, Y. Chen and J. Wang, Polymers, 2015, 7, 909-920.

37 J. Li, L. Zhang, J. Gu, Y. Sun and X. Ji, RSC Adv., 2015, 5, 19859-19864.

38 S. Gou, Q. Zhang, C. Yang, Q. Li, S. Xu, Y. Wu and Q. Guo, RSC Adv., 2016, 6, 76696-76706.

39 X. Li, H. Zheng, Y. Wang, Y. Sun, B. Xu and C. Zhao, Chem. Eng. J., 2017, 319, 119-130.

40 S. Gou, S. Li, M. Feng, Q. Zhang, Q. Pan, J. Wen, Y. Wu and Q. Guo, Ind. Eng. Chem. Res., 2017, 56, 942-951.

41 A. Pourjavadi, S. M. Fakoorpoor and S. H. Hosseini, Carbohydr. Polym., 2013, 93, 506-511.

42 Z. Abdollahi, M. Frounchi and S. Dadbin, J. Ind. Eng. Chem., 2011, 17, 580-586.

43 J. Li, C. Hu, J. Shao, H. Li, P. Li, X. Li and W. He, Polymer, 2017, 119, 152-159.

44 Z. L. Yang, B. Y. Gao, C. X. Li, Q. Y. Yue and B. Liu, Chem. Eng. J., 2010, 161, 27-33.

45 H. Zheng, J. Ma, C. Zhu, Z. Zhang, L. Liu, Y. Sun and X. Tang, Sep. Purif. Technol., 2014, 123, 35-44.

46 H. Zheng, Y. Sun, J. Guo, F. Li, W. Fan, Y. Liao and Q. Guan, Ind. Eng. Chem. Res., 2014, 53, 2572-2582.
47 Z. Yang, K. Ren, E. Guibal, S. Jia, J. Shen, X. Zhang and W. Yang, Chemosphere, 2016, 161, 482-490.

48 Z. Yang, Y. Shang, Y. Lu, Y. Chen, X. Huang, A. Chen, Y. Jiang, W. Gu, X. Qian, H. Yang and R. Cheng, Chem. Eng. J., 2011, 172, 287-295.

49 Z. Yang, H. Yang, Z. Jiang, T. Cai, H. Li, H. Li, A. Li and R. Cheng, J. Hazard. Mater., 2013, 255, 36-45.

50 L. You, L. Song, A. Wang, F. Lu and Q. Zhang, Colloids Surf., A, 2017, 520, 841-849.

51 M. K. R. Konduri and P. Fatehi, Colloids Surf., A, 2017, 530, 20-32.

52 C. Zhao, H. Zheng, B. Gao, Y. Liu, J. Zhai, S. Zhang and B. Xu, Ultrason. Sonochem., 2018, 42, 31-41.

53 J. Ma, K. Fu, X. Fu, Q. Guan, L. Ding, J. Shi, G. Zhu, X. Zhang, S. Zhang and L. Jiang, Sep. Purif. Technol., 2017, 182, 134143.

54 Y. X. Zhao, B. Y. Gao, H. K. Shon, J. H. Kim, Q. Y. Yue and Y. Wang, Sep. Purif. Technol., 2011, 81, 332-338.

55 R. Mao, Y. Wang, B. Zhang, W. Xu, M. Dong and B. Gao, Desalination, 2013, 314, 161-168.

56 Y. Adachi, L. Feng and M. Kobayashi, Colloids Surf., A, 2015, 471, 38-44.

57 A. Tironi, M. A. Trezza, E. F. Irassar and A. N. Scian, Procedia Mater. Sci., 2012, 1, 343-350.

58 B. Y. Du, Z. Cao, Z. B. Li, A. X. Mei, X. H. Zhang, J. J. Nie, J. T. Xu and Z. Q. Fan, Langmuir, 2009, 25, 12367-12373. 